Karl-Franzens Universität Graz

Technische Universität Graz

Medizinische Universität Graz

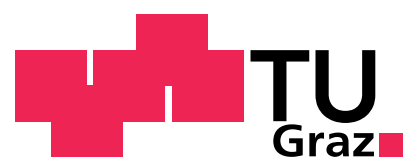

\title{
Optimal control of the bidomain system (I): The monodomain approximation with the Rogers-McCulloch model
}

\author{
Marcus Wagner Karl Kunisch
}


SFB sponsors:

- Austrian Science Fund (FWF)

- University of Graz

- Graz University of Technology

- Medical University of Graz

- Government of Styria

- City of Graz

Das Land

Steiermark

Stadt $\mathbf{G} \mathbf{R}$ A Z $\mathbf{Z}$ Wissenschaft 


\title{
Optimal control of the bidomain system (I): The monodomain approximation with the Rogers-McCulloch model. Revised version
}

\author{
Karl Kunisch and Marcus Wagner
}

\section{Introduction.}

The present work opens a series of papers where we will set forth a basic framework for optimal control of the bidomain equations together with related uniqueness and regularity results. ${ }^{01)}$ The full bidomain system, which represents a well-established description of the electrical activity of the heart, is given by

$$
\begin{aligned}
& \frac{\partial \Phi}{\partial t}+I_{i o n}(\Phi, W)-\operatorname{div}\left(M_{i} \nabla \Phi_{i}\right)=\quad I_{i} \quad \text { for a. a. }(x, t) \in \Omega \times[0, T] ; \\
& \frac{\partial \Phi}{\partial t}+I_{i o n}(\Phi, W)+\operatorname{div}\left(M_{e} \nabla \Phi_{e}\right)=-I_{e} \text { for a. a. }(x, t) \in \Omega \times[0, T] ; \\
& \frac{\partial W}{\partial t}+G(\Phi, W) \quad=0 \text { for a. a. }(x, t) \in \Omega \times[0, T] \text {; } \\
& \mathfrak{n}^{\mathrm{T}} M_{i} \nabla \Phi_{i}=0 \text { and } \mathfrak{n}^{\mathrm{T}} M_{e} \nabla \Phi_{e}=0 \text { for all }(x, t) \in \partial \Omega \times[0, T] ; \\
& \Phi(x, 0)=\Phi_{0}(x) \text { and } W(x, 0)=W_{0}(x) \text { for a. a. } x \in \Omega,
\end{aligned}
$$

together with appropriate specifications of the ionic current $I_{i o n}$ and the function $G$ within the gating equation (1.3). ${ }^{02)}$ Within the cardiac muscle, which occupies the spatial domain $\Omega \subset \mathbb{R}^{3}$, the anisotropic properties of the intracellular and extracellular tissue parts will be described by conductivity tensors $M_{i}$ and $M_{e}$. The variables $\Phi_{i}=\Phi_{i}(x, t)$ and $\Phi_{e}=\Phi_{e}(x, t)$ represent the intracellular and extracellular electrical potential; their difference $\Phi=\Phi_{i}-\Phi_{e}$ is the transmembrane potential. Further, $I_{i}$ and $I_{e}$ model the intracellular and extracellular stimulation current, respectively. $W$, the so-called gating variable, is related to the ion transport through the cell membrane. On a microscopical level, the intracellular and extracellular quantities are concentrated on disjoint subdomains $\Omega_{i}$ and $\Omega_{e}$ of $\Omega$, whose common boundary represents the total of the cell membranes. ${ }^{03)}$ After an averaging procedure, ${ }^{04)}$ the macroscopic model $(1.1)-(1.5)$ is obtained, where the superimposed intracellular and extracellular media occupy the same domain $\Omega$.

The present paper is concerned with the monodomain equations, which arise from $(1.1)-(1.5)$ as a special case if the conductivity tensors satisfy $M_{e}=\lambda M_{i}$ with a constant parameter $\lambda>0$. Then $\Phi_{e}$ can be eliminated from $(1.1)-(1.5)$, and we get the monodomain system

$$
\begin{aligned}
& \frac{\partial \Phi}{\partial t}+I_{i o n}(\Phi, W)-\frac{\lambda}{1+\lambda} \operatorname{div}\left(M_{i} \nabla \Phi\right)=\frac{1}{1+\lambda}\left(\lambda I_{i}-I_{e}\right) \quad \text { for a. a. }(x, t) \in \Omega \times[0, T] ; \\
& \frac{\partial W}{\partial t}+G(\Phi, W)=0 \quad \text { for a. a. }(x, t) \in \Omega \times[0, T] ; \\
& \mathfrak{n}^{\mathrm{T}} M_{i} \nabla \Phi=0 \text { for all }(x, t) \in \partial \Omega \times[0, T] ; \\
& \Phi(x, 0)=\Phi_{0}(x) \text { and } W(x, 0)=W_{0}(x) \quad \text { for a. a. } x \in \Omega
\end{aligned}
$$

01) For an introduction to PDE-constrained optimal control problems, cf. [ITo/Kunisch 08] and [TRÖLTZSCH 09] .

02) First considered in [Tung 78]. For a more detailed introduction to the model, we refer to [SUNDNES/LinES/CAI/ Nielsen/MARDAl/TVEito 06], pp. $21-56$, and the references therein.

03) See [Colli Franzone/Savaré 02], pp. 49-52, and [Veneroni 06].

04) Described in [Colli Franzone/Savaré 02], pp. $71-75$. 
as a considerable simplification of (1.1) - (1.5) which, nevertheless, conserves some essential features of the full bidomain model as excitability phenomena. For this reason, this system has deserved noticeable attention for itself. ${ }^{05)}$

The focus of the present paper is the investigation of the following optimal control problem:

(P) $F\left(\Phi, W, I_{e}\right)=\int_{0}^{T} \int_{\Omega} r(x, t, \Phi(x, t), W(x, t)) d x d t+\frac{\mu}{2} \int_{0}^{T} \int_{\Omega} I_{e}(x, t)^{2} d x d t \longrightarrow \inf$ !

subject to the state equations $(1.6)-(1.9)$ in its weak formulation (see (2.1) - (2.3) below) and

the control restriction $\left|I_{e}(x, t)\right| \leqslant R$ for a. a. $(x, t) \in \Omega \times[0, T]$

with $R>0$ and a function $r(x, t, \varphi, w)$ to be specified below. In this problem, the monodomain system will be controlled by means of the excitation variables on the right-hand side of (1.6). Since in practical situations the application of an excitation to the intracellular part of the tissue is impossible, we have $I_{i}=\mathfrak{o}$, and the single control variable is $I_{e}$. The control restriction (1.11) reflects the obvious fact that one cannot apply arbitrary large electrical stimulations to living tissue without damaging it.

For several reasons, it is appropriate to rely on a weak solution concept for the state equations in the context of optimal control. First, typical control functions are nonsmooth (in fact, we will work with $I_{e} \in$ $\left.L^{\infty}\left[(0, T), L^{2}(\Omega)\right]\right)$; consequently, the corresponding state variables $\Phi$ and $W$ will fit into Sobolev spaces rather than in spaces of continously differentiable functions. Second, the existence proof for global and local minimizers requires the closedness of the feasible domain in a suitable weak topology. The necessary optimality conditions are stated and proved within reflexive spaces as well. Thus the monodomain system will be included into $(\mathrm{P})$ in its weak formulation, dispensing with a further regularity analysis of the solutions. For the first member within the objective (1.10), a typical choice is a tracking-type integrand $r=\frac{1}{2}(\varphi-$ $\left.\Phi_{s t}(x)\right)^{2}$ where $\Phi_{s t}$ is taken, for example, from a steady-state solution $\left(\Phi_{s t}, W_{s t}\right)$ of $(1.6)-(1.9)$. The second summand constitutes the control costs. Its interpretation is that solutions with little intervention into the cardiac system should be favored.

The main result of the present paper is the rigorous proof of the following set of first-order necessary optimality conditions for weak local minimizers $\left(\hat{\Phi}, \hat{W}, \hat{I}_{e}\right)$ of $(\mathrm{P})$, consisting of the variational inequality

$$
\int_{0}^{T} \int_{\Omega}\left(\mu \hat{I}_{e}(x, t)+\frac{1}{1+\lambda} P_{1}(x, t)\right)\left(I_{e}(x, t)-\hat{I}_{e}(x, t)\right) d x d t \geqslant 0 \text { for all feasible controls } I_{e}
$$

and the adjoint system

$$
\begin{aligned}
& -\frac{\partial P_{1}}{\partial t}-\nabla \cdot\left(\frac{\lambda}{1+\lambda} M_{i} \nabla P_{1}\right)+\frac{\partial I_{i o n}}{\partial \varphi}(\hat{\Phi}, \hat{W}) P_{1}=-\frac{\partial G}{\partial \varphi}(\hat{\Phi}, \hat{W}) P_{2}-\frac{\partial r}{\partial \varphi}(\hat{\Phi}, \hat{W}) \text { for a. a. }(x, t) \in \Omega_{T} \\
& \mathfrak{n}^{\mathrm{T}} M_{i} \nabla P_{1}=0 \text { for all }(x, t) \in \partial \Omega \times[0, T] ; \quad P_{1}(x, T)=0 \text { for a. a. } x \in \Omega \\
& -\frac{\partial P_{2}}{\partial t}+\frac{\partial G}{\partial w}(\hat{\Phi}, \hat{W}) P_{2}=-\frac{\partial I_{i o n}}{\partial w}(\hat{\Phi}, \hat{W}) P_{1}-\frac{\partial r}{\partial w}(\hat{\Phi}, \hat{W}) \text { for a. a. }(x, t) \in \Omega_{T} \\
& P_{2}(x, T)=0 \text { for a. a. } x \in \Omega
\end{aligned}
$$

for the multipliers $P_{1}$ and $P_{2}$ related to (1.6) and (1.7), respectively (see Theorem 3.5. below). Treating (P) as a weakly singular problem in the sense of ITO/KUNISCH, ${ }^{06)}$ the proof of $(1.12)-(1.16)$ requires two main

05) See e. g. [PAO 92 ], pp. 681 ff., and the literature cited therein (p. 745).

06) [ITO/Kunisch 08], p. $17 \mathrm{f}$. 
ingredients: a stability estimate for the state equations (Theorem 3.8.), and an existence theorem for the adjoint equations (Theorem 3.9.).

Although the bidomain system has been extensively studied under computational aspects, ${ }^{07)}$ only little work related to its optimal control is available in the literature as yet. Problem (1.10) - (1.11) was already considered in [NAGaiah/Kunisch/Plank 09] and [NAGAiah/Kunisch 11] . In these papers, the control problem has been successfully numerically accessed on the base of gradient or inexact Newton techniques, respectively, but the optimality system has been derived only formally without proof. [AinseBa/Bendahmane/ RUIZ-BAIER 10] study an optimal control problem on a tridomain model. Even here, the optimality conditions have been derived only formally. Another related control problem was investigated in [BRANDÃO/FeRNÁNDEZ-CARA/MAGALHÃEs/RoJAS-MEDAR 08] where the authors study a tracking-type functional, restricting themselves in (1.6) - (1.9) to the FitzHugh-Nagumo model for $I_{i o n}$ and $G$ and replacing (1.8) by Dirichlet boundary conditions. In this particular case, the authors obtain necessary optimality conditions by means of the Dubovitskij-Milyutin formalism. In the context of defibrillation, [MUZDEKA/BARBIERI 05] pursued a different approach. After disregarding the nonlinearities, the authors perform a spectral approximation and solve a time-optimal control problem for the ODE, which arises for the lumped mass system resulting from the eigenmode expansion.

The structure of the paper is as follows. In Section 2, we summarize existence and uniqueness results for weak solutions to (1.5) - (1.9) for the Rogers-McCulloch, FitzHugh-Nagumo and the linearized Aliev-Panfilov model. In Section 3, we turn to the study of the optimal control problem (P). After restating the problem within function spaces and confirming the existence of minimizers, we describe the adjoint system and the optimality conditions. The main part of the section is devoted to the proof of the first-order necessary conditions. Throughout the paper, the proofs will be worked out for the Rogers-McCulloch model. Note that, nevertheless, they do remain valid for the FitzHugh-Nagumo and the linearized Aliev-Panfilov model. The corresponding alterations will be indicated. For the conveniency of the reader, we collect some facts about Bochner integrable mappings in an appendix (Section 4). A further appendix (Section 5) contains the proof of the stability estimate for the monodomain system (Theorem 3.8.).

\section{Notations.}

We denote by $L^{p}(\Omega)$ the space of functions, which are in the $p$ th power integrable $(1 \leqslant p<\infty)$, or are measurable and essentially bounded $(p=\infty)$, and by $W^{1, p}(\Omega)$ the Sobolev space of functions $\psi: \Omega \rightarrow \mathbb{R}$ which, together with their first-order weak partial derivatives, belong to the space $L^{p}(\Omega, \mathbb{R})(1 \leqslant p<\infty)$. For spaces of Bochner integrable mappings, e. g. $L^{2}\left[(0, T), W^{1,2}(\Omega)\right]$, we refer to Section $4 . \Omega_{T}$ is an abbreviation for $\Omega \times[0, T]$. The gradient $\nabla$ is always taken only with respect to the spatial variables $x$. The abbreviation " $(\forall) t \in \mathrm{A}$ " has to be read as "for almost all $t \in \mathrm{A}$ " or "for all $t \in \mathrm{A}$ except a Lebesgue null set", and the symbol $\mathfrak{o}$ denotes, depending on the context, the zero element or the zero function of the underlying space.

07) We refer e. g. to [Colli Franzone/Deuflhard/Erdmann/Lang/Pavarino 06], [Vigmond/Aguel/TrayaNOva 02 ] and [Weber dos Santos/Plank/Bauer/Vigmond 04]. 


\section{Weak solutions of the monodomain system.}

a) Weak formulation of the monodomain system.

Let $\Omega \subset \mathbb{R}^{3}$ be a bounded, open set, and $T>0$. The weak formulation of the monodomain system (1.6) - (1.9), on which the formulation of the optimal control problem in Section 3 will be based, reads as follows:

$$
\begin{aligned}
& \int_{\Omega}\left(\frac{\partial \Phi}{\partial t}+I_{\text {ion }}(\Phi, W)\right) \psi d x+\int_{\Omega} \frac{\lambda}{1+\lambda} \nabla \psi^{\mathrm{T}} M_{i} \nabla \Phi d x=\int_{\Omega} \frac{1}{1+\lambda}\left(\lambda I_{i}-I_{e}\right) \psi d x \\
& \forall \psi \in W^{1,2}(\Omega)(\forall) t \in[0, T] ; \\
& \int_{\Omega}\left(\frac{\partial W}{\partial t}+G(\Phi, W)\right) \psi d x=0 \quad \forall \psi \in L^{2}(\Omega)(\forall) t \in[0, T] ; \\
& \Phi(x, 0)=\Phi_{0}(x) \quad(\forall) x \in \Omega ; \quad W(x, 0)=W_{0}(x) \quad(\forall) x \in \Omega . \\
& \text { finition 2.1. (Weak solution of the monodomain system) }{ }^{08)} \text { A pair }(\Phi, W) \text { is called a weak } \\
& \text { distributional sense and obey the initial conditions. Moreover, the functions belong to the spaces } \\
& \Phi \in C^{0}\left[[0, T], L^{2}(\Omega)\right] \cap L^{2}\left[(0, T), W^{1,2}(\Omega)\right] \cap L^{p}\left(\Omega_{T}\right) \quad \text { with } 2 \leqslant p \leqslant 6 ; \\
& W \in C^{0}\left[[0, T], L^{2}(\Omega)\right] .
\end{aligned}
$$

Definition 2.1. (Weak solution of the monodomain system) ${ }^{08)}$ A pair $(\Phi, W)$ is called a weak solution of the monodomain system $(2.1)-(2.3)$ on $[0, T]$ iff $\Phi$ and $W$ satisfy the equations on $[0, T]$ in the distributional sense and obey the initial conditions. Moreover, the functions belong to the spaces

\section{b) Models for the ionic current.}

The ionic current through the cell membranes will be described with the help of a so-called gating variable $W$, which is coupled with the transmembrane voltage $\Phi$ by an ODE. We will consider the following models:

a) The Rogers-McCulloch model. ${ }^{09)}$

$$
\begin{aligned}
& I_{\text {ion }}(\varphi, w)=b \cdot \varphi(\varphi-a)(\varphi-1)+\varphi \cdot w=b \varphi^{3}-(a+1) b \varphi^{2}+a b \varphi+\varphi w \\
& G(\varphi, w)=\varepsilon w-\varepsilon \kappa \varphi
\end{aligned}
$$

with $0<a<1, b>0, \kappa>0$ and $\varepsilon>0$. Consequently, the gating variable obeys the linear ODE

$$
\frac{\partial W}{\partial t}+\varepsilon W=\varepsilon \kappa \Phi
$$

b) The FitzHugh-Nagumo model. ${ }^{10)}$

$$
\begin{aligned}
& I_{i o n}(\varphi, w)=\varphi(\varphi-a)(\varphi-1)+w=\varphi^{3}-(a+1) \varphi^{2}+a \varphi+w ; \\
& G(\varphi, w)=\varepsilon w-\varepsilon \kappa \varphi
\end{aligned}
$$

with $0<a<1, \kappa>0$ and $\varepsilon>0$. Consequently, the gating variable obeys the same linear ODE (2.8) as before.

08) See [Bourgault/Coudière/Pierre 09] , p. 472, Definition 26.

09) [Rogers/MCCULLOCH 94].

10) [FitzHugh 61], together with [Nagumo/Arimoto/Yoshizawa 62]. 
c) The linearized Aliev-Panfilov model. ${ }^{11)}$

$$
\begin{aligned}
& I_{i o n}(\varphi, w)=b \cdot \varphi(\varphi-a)(\varphi-1)+\varphi \cdot w=b \varphi^{3}-(a+1) b \varphi^{2}+a b \varphi+\varphi w ; \\
& G(\varphi, w)=\varepsilon w-\varepsilon \kappa\left((a+1) \varphi-\varphi^{2}\right)
\end{aligned}
$$

with $0<a<1, b>0, \kappa>0$ and $\varepsilon>0$. The linear ODE for the gating variable is

$$
\frac{\partial W}{\partial t}+\varepsilon W=\varepsilon \kappa\left((a+1) \Phi-\Phi^{2}\right) .
$$

\section{c) Existence and uniqueness of weak solutions.}

There are different ways to prove existence and uniqueness results for the monodomain system. In the context of smooth solutions, the upper-lower-solution techniques applied in [PAO 92 ], pp. $681 \mathrm{ff}$., could be extended to the Rogers-McCulloch model. Another possibility would be the specialization of results on the bidomain system, but then one has to impose additional conditions on the spectral properties of the conductivity tensors. ${ }^{12)}$ In order to get weak solvability in reflexive spaces without additional assumptions about $M_{i}$ and $M_{e}$, we pursue the approach of [Bourgault/Coudière/Pierre 09] for the existence part only and base the uniqueness result on the stability estimate (Theorem 3.8.), which is part of the proof of the necessary optimality conditions. Note that the monodomain system is solvable without the compatibility condition $\int_{\Omega}\left(I_{i}(x, t)+I_{e}(x, t)\right) d x=0(\forall) t \in(0, T)$, which is a mandatory assumption in the full bidomain case.

Theorem 2.2. (Existence of weak solutions) ${ }^{13)}$ Assume that the data within (2.1) - (2.3) obey the following assumptions:

a) $\Omega \subset \mathbb{R}^{3}$ is a bounded Lipschitz domain.

b) $M_{i}: \operatorname{cl}(\Omega) \rightarrow \mathbb{R}^{3 \times 3}$ is a symmetric, positive definite matrix function with $L^{\infty}(\Omega)$-coefficients, which obeys a uniform ellipticity condition with $\mu_{1}, \mu_{2}>0$ :

$$
0 \leqslant \mu_{1}\|\xi\|^{2} \leqslant \xi^{\mathrm{T}} M_{i}(x) \xi \leqslant \mu_{2}\|\xi\|^{2} \quad \forall \xi \in \mathbb{R}^{3} \quad \forall x \in \Omega
$$

Then for any of the models from Subsection 2.b), the monodomain system (2.1) - (2.3) admits for arbitrary initial values $\Phi_{0}, W_{0} \in L^{2}(\Omega)$ and inhomogeneities $I_{i}, I_{e} \in L^{2}\left[(0, T),\left(W^{1,2}(\Omega)\right)^{*}\right]$ at least one weak solution $(\Phi, W)$ in the sense of Definition 2.1. with $p=4$.

Proof. Observe first that the reformulated bidomain system in [Bourgault/Coudière/Pierre 09], p. 473 , Lemma 28 , and the monodomain system $(2.1)-(2.3)$ have the same structure. In the monodomain system, however, the bilinear form $M: W^{1,2}(\Omega) \times W^{1,2}(\Omega) \rightarrow \mathbb{R}$ reads as

$$
M\left(\psi_{1}, \psi_{2}\right)=\frac{\lambda}{1+\lambda} \int_{\Omega} \nabla \psi_{1}^{\mathrm{T}} M_{i} \nabla \psi_{2} d x .
$$

Lemma 2.3. ${ }^{14)}$ The bilinear form $M$ is symmetric, continuous and coercive, satisfying with $\beta, \gamma>0$

$$
\begin{aligned}
& \beta\|\psi\|_{W^{1,2}(\Omega)}^{2} \leqslant M(\psi, \psi)+\beta\|\psi\|_{L^{2}(\Omega)}^{2} \quad \forall \psi \in W^{1,2}(\Omega) \quad \text { and } \\
& \left|M\left(\psi_{1}, \psi_{2}\right)\right| \leqslant \gamma \cdot\left\|\psi_{1}\right\|_{W^{1,2}(\Omega)} \cdot\left\|\psi_{2}\right\|_{W^{1,2}(\Omega)} \quad \forall \psi_{1}, \psi_{2} \in W^{1,2}(\Omega) .
\end{aligned}
$$

11) See [Aliev/Panfilov 96]. The linearized model is taken from [Bourgault/Coudière/PierRe 09], p. 480. Instead, the original model contains a Riccati equation for the gating variable.

12) Cf. [Boulakia/Fernández/Gerbeau/Zemzemi 08], p. 8, (2.18), and [Bourgault/Coudière/Pierre 09], p. 478 f., Theorem 32 .

13) Slightly modified from [Bourgault/Coudière/Pierre 09] , p. 473, Theorem 30.

14) Compare with [Bourgault/Coudière/Pierre 09], p. 464, Theorem 6. 
Proof. As a consequence of Assumption b), we have

$$
\begin{array}{r}
\frac{\lambda \mu_{1}}{1+\lambda} \int_{\Omega}|\nabla \psi|^{2} d x \leqslant \frac{\lambda}{1+\lambda} \int_{\Omega} \nabla \psi^{\mathrm{T}} M_{i} \nabla \psi d x=M(\psi, \psi) \quad \forall \psi \in W^{1,2}(\Omega) \\
\frac{\lambda \mu_{1}}{1+\lambda}\|\psi\|_{W^{1,2}(\Omega)}^{2} \leqslant M(\psi, \psi)+\frac{\lambda \mu_{1}}{1+\lambda}\|\psi\|_{L^{2}(\Omega)}^{2} \quad \forall \psi \in W^{1,2}(\Omega) .
\end{array}
$$

The uniform ellipticity of $M_{i}$ implies the second inequality as well.

Obviously, the form $M$ generates a weak operator on $W^{1,2}(\Omega) \times W^{1,2}(\Omega)$. Consequently, the existence proof from [Bourgault/Coudière/Pierre 09], pp. 473 ff., Subsections 5.2.1. - 5.3. can be carried over to (2.1) - (2.3) after replacing the bidomain bilinear form by $M$.

Theorem 2.4. (A priori estimate for weak solutions) Under the assumptions of Theorem 2.2., specifying any of the models from Subsection 2.b), consider a pair

$(\Phi, W) \in\left(C^{0}\left[[0, T], L^{2}(\Omega)\right] \cap L^{2}\left[(0, T), W^{1,2}(\Omega)\right] \cap L^{p}\left(\Omega_{T}\right)\right) \times C^{0}\left[[0, T], L^{2}(\Omega)\right]$,

which forms a weak solution of the monodomain system $(2.1)-(2.3)$ on $[0, T]$ with $p=4$. Then the following estimate holds:

$$
\begin{array}{r}
\|\Phi\|_{C^{0}\left[[0, T], L^{2}(\Omega)\right]}^{2}+\|\Phi\|_{L^{2}\left[(0, T), W^{1,2}(\Omega)\right]}^{2}+\|\Phi\|_{L^{p}\left(\Omega_{T}\right)}^{p}+\|\partial \Phi / \partial t\|_{L^{q}\left[(0, T),\left(W^{1,2}(\Omega)\right)^{*}\right]}^{q} \\
+\|W\|_{C^{0}\left[[0, T], L^{2}(\Omega)\right]}^{2}+\|\partial W / \partial t\|_{L^{2}\left[(0, T),\left(W^{1,2}(\Omega)\right)^{*}\right]}^{2} \\
\leqslant C \cdot\left(1+\left\|\Phi_{0}\right\|_{L^{2}(\Omega)}^{2}+\left\|W_{0}\right\|_{L^{2}(\Omega)}^{2}+\left\|I_{i}\right\|_{L^{2}\left[(0, T),\left(W^{1,2}(\Omega)\right)^{*}\right]}^{2}+\left\|I_{e}\right\|_{L^{2}\left[(0, T),\left(W^{1,2}(\Omega)\right)^{*}\right]}^{2}\right)(2.20)
\end{array}
$$

where $p=4$ and $q=4 / 3$. The constant $C>0$ does not depend on $\Phi_{0}, W_{0}, I_{i}$ and $I_{e}$.

Proof. For the same reasons as in the proof of Theorem 2.2., the arguments from [NAGAIAH/KunISCH/ Plank 09 ] , p. 10 f., Lemma 3.5., as well as the underlying estimates from [Bourgault/Coudière/PiERRE 09 ], pp. $474-476$, may be carried over to the monodomain system $(2.1)-(2.3)$.

Theorem 2.5. (Uniqueness of weak solutions) Assume that the data within (2.1) - (2.3) obey the assumptions of Theorem 2.2., and specify within the system any of the models from Subsection 2.b). Then for arbitrary initial values $\Phi_{0} \in L^{2}(\Omega), W_{0} \in L^{4}(\Omega)$ and inhomogeneities $I_{i}, I_{e} \in L^{\infty}\left[(0, T),\left(W^{1,2}(\Omega)\right)^{*}\right]$, the monodomain system $(2.1)-(2.3)$ admits on $[0, T]$ a unique weak solution $(\Phi, W)$ in the sense of Definition 2.1. with $p=4$.

Proof. In order to confirm uniqueness, apply Theorem 3.8. below to $I_{i}{ }^{\prime}=I_{i}{ }^{\prime \prime}=I_{i}$ and $I_{e}{ }^{\prime}=I_{e}{ }^{\prime \prime}=I_{e}$. 


\section{Optimal control problems for the monodomain system.}

\section{a) Statement of the control problem.}

It will be convenient to restate the optimal control problem $(\mathrm{P})$ within an appropriate function space framework. Let us consider the spaces

$$
\mathrm{X}_{1}=L^{2}\left[(0, T), W^{1,2}(\Omega)\right], \quad \mathrm{X}_{2}=L^{2}\left[(0, T), L^{2}(\Omega)\right], \quad \mathrm{X}_{3}=L^{\infty}\left[(0, T), L^{2}(\Omega)\right],
$$

the subspaces

$$
\begin{aligned}
& \widetilde{\mathrm{X}}_{1}=\mathrm{X}_{1} \cap W^{1,4 / 3}\left[(0, T),\left(W^{1,2}(\Omega)\right)^{*}\right] \cap C^{0}\left[[0, T], L^{2}(\Omega)\right], \\
& \widetilde{\mathrm{X}}_{2}=\mathrm{X}_{2} \cap W^{1,2}\left[(0, T),\left(L^{2}(\Omega)\right)^{*}\right] \cap C^{0}\left[[0, T], L^{2}(\Omega)\right],
\end{aligned}
$$

which contain all polynomials and, consequently, lie dense in $\mathrm{X}_{1}$ and $\mathrm{X}_{2}$, and the target spaces

$$
\mathrm{Z}_{1}=L^{4 / 3}\left[(0, T),\left(W^{1,2}(\Omega)\right)^{*}\right], \quad \mathrm{Z}_{2}=L^{2}\left[(0, T),\left(L^{2}(\Omega)\right)^{*}\right], \quad \mathrm{Z}_{3}=\mathrm{Z}_{4}=L^{2}(\Omega) .
$$

With the aid of operators $F: \mathrm{X}_{1} \times \mathrm{X}_{2} \times \mathrm{X}_{3} \rightarrow \mathbb{R}, E_{1}: \widetilde{\mathrm{X}}_{1} \times \widetilde{\mathrm{X}}_{2} \times \mathrm{X}_{3} \rightarrow \mathrm{Z}_{1}, E_{2}: \widetilde{\mathrm{X}}_{1} \times \widetilde{\mathrm{X}}_{2} \rightarrow \mathrm{Z}_{2}$, $E_{3}: \widetilde{\mathrm{X}}_{1} \rightarrow \mathrm{Z}_{3}$ and $E_{4}: \widetilde{\mathrm{X}}_{2} \rightarrow \mathrm{Z}_{4}$, the optimal control problem $(\mathrm{P})$ can be written as follows:

(P) $\quad F\left(\Phi, W, I_{e}\right)=\int_{0}^{T} \int_{\Omega} r(x, t, \Phi(x, t), W(x, t)) d x d t+\frac{\mu}{2} \int_{0}^{T} \int_{\Omega} I_{e}(x, t)^{2} d x d t \longrightarrow \inf ! ;$

$$
E_{1}\left(\Phi, W, I_{e}\right)=\mathfrak{o} \Longleftrightarrow \int_{\Omega}\left(\frac{\partial \Phi(t)}{\partial t}+I_{\text {ion }}(\Phi(t), W(t))+\frac{1}{1+\lambda} I_{e}(t)\right) \psi d x
$$

$$
+\frac{\lambda}{1+\lambda} \int_{\Omega} \nabla \psi^{\mathrm{T}} M_{i} \nabla \Phi(t) d x=0 \quad \forall \psi \in W^{1,2}(\Omega)(\forall) t \in[0, T] ;
$$

$E_{2}(\Phi, W)=\mathfrak{o} \Longleftrightarrow \int_{\Omega}\left(\frac{\partial W(t)}{\partial t}+G(\Phi(t), W(t))\right) \psi d x=0 \quad \forall \psi \in L^{2}(\Omega)(\forall) t \in[0, T] ;$

$E_{3}(\Phi)=\mathfrak{o} \quad \Longleftrightarrow \Phi(x, 0)-\Phi_{0}(x)=0 \quad(\forall) x \in \Omega ;$

$E_{4}(W)=\mathfrak{o} \Longleftrightarrow W(x, 0)-W_{0}(x)=0 \quad(\forall) x \in \Omega ;$

$I_{e} \in \mathcal{C}=\left\{Z \in L^{\infty}\left[(0, T), L^{2}(\Omega)\right]|| Z(x, t) \mid \leqslant R(\forall)(x, t) \in \Omega_{T}\right\}$.

Concerning the data, we take the assumptions of Theorem 2.2. The numbers $T>0, \lambda>0, \mu>0$ and $R>0$ as well as the functions $\Phi_{0} \in L^{2}(\Omega)$ and $W_{0} \in L^{4}(\Omega)$ are fixed. The functions $I_{i o n}$ and $G$ are specified according to the Rogers-McCulloch model. Then by Theorem 2.5., the control-to-state mapping $\mathcal{C} \ni I_{e} \longmapsto\left(\Phi\left(I_{e}\right), W\left(I_{e}\right)\right)$ is well-defined. The spaces in (3.1) - (3.4) have been chosen with regard to Theorems 2.2. and 2.4.

\section{b) Structure of the feasible domain and existence of global minimizers.}

Proposition 3.1. The set $\mathcal{C}$ of the admissible controls according to (3.10) forms a closed, convex, weak*sequentially compact subset of $L^{\infty}\left[(0, T), L^{2}(\Omega)\right]$.

Proof. The convexity of $\mathcal{C}$ is obvious. In order to prove closedness, consider a sequence $\left\{Z^{N}\right\}, L^{\infty}[(0, T)$, $\left.L^{2}(\Omega)\right]$, which converges in norm to a limit element $\hat{Z}$. Then there exists a subsequence, which converges a. e. pointwise on $\Omega_{T}$ to $\hat{Z}$, and the limit element obeys the a. e. pointwise restriction as well. Then the weak*-sequential compactness follows from [RoLEwICZ 76] , p. 301, Theorem VI.6.6., together with p. 152, Theorem IV.4.11. 
Proposition 3.2. Under the assumptions from Subsection 3.a), the feasible domain $\mathcal{B}$ of the problem $(\mathrm{P})$ is nonempty and closed with respect to the following topology in $\mathrm{X}_{1} \times \mathrm{X}_{2} \times \mathrm{X}_{3}$ : weak convergence with respect to the first and second component, weak ${ }^{*}$-convergence with respect to the third component.

Proof. The existence of feasible solutions for $(\mathrm{P})$ follows from Theorem 2.2. Assume that a sequence $\left\{\left(\Phi^{N}, W^{N}, I_{e}^{N}\right)\right\}, \mathcal{B}$ with $\Phi^{N} \longrightarrow \mathrm{X}_{1} \hat{\Phi}, W^{N} \longrightarrow \mathrm{X}_{2} \hat{W}$ and $I_{e}^{N} \stackrel{*}{\mathrm{X}_{3}} \hat{I}_{e}$ is given. Then, by Proposition 3.1., $\hat{I}_{e}$ belongs to $\mathcal{C}$. Theorem 2.4. yields uniform bounds for the norms of $\Phi^{N}$ and $W^{N}$, which imply weak convergence of $\partial \Phi^{N^{\prime}} / \partial t, \nabla \Phi^{N^{\prime}}$ and $\partial W^{N^{\prime}} / \partial t$ as well as a. e. pointwise convergence of $\Phi^{N^{\prime}}$ on $\Omega_{T}$ along a suitable subsequence. Consequently, passing to the limit in $(2.1)-(2.3)$, we see that $(\hat{\Phi}, \hat{W})$ solves the monodomain system with right-hand side $\hat{I}_{e} \cdot{ }^{15}$

Theorem 3.3. (Existence of global minimizers) Assume that the integrand $r(x, t, \varphi, w): \Omega \times[0, T] \times$ $\mathbb{R} \times \mathbb{R}$ is bounded from below, measurable with respect to $x$ and $t$ and continuous and convex with respect to $\varphi$ and $w$. Then under the assumptions from Subsection 3.a), the problem (P) admits a global minimizer.

Proof. Together with $r$, the objective $F$ is bounded from below, and the problem $(\mathrm{P})$ admits a minimizing sequence $\left\{\left(\Phi^{N}, W^{N}, I_{e}{ }^{N}\right)\right\}, \mathcal{B}$. Since $\left\|I_{e}{ }^{N}\right\|_{\mathrm{X}_{3}}$ is uniformly bounded, Theorem 2.4. implies the boundedness of $\left\|\Phi^{N}\right\|_{\mathrm{X}_{1}}$ and $\left\|W^{N}\right\|_{\mathrm{X}_{2}}$ as well, and we may pass to a subsequence $\left\{\left(\Phi^{N^{\prime}}, W^{N^{\prime}}, I_{e}^{N^{\prime}}\right)\right\}$ with $\Phi^{N^{\prime}} \rightarrow \hat{\Phi}$, $W^{N^{\prime}} \rightarrow \hat{W}$ and $I_{e}{ }^{N^{\prime}} \stackrel{*}{\longrightarrow} \hat{I}_{e}$. By Proposition 3.2., $\left(\hat{\Phi}, \hat{W}, \hat{I}_{e}\right)$ is feasible. The lower semicontinuity of the objective may be confirmed as in [DAcorognA 08], p. 96, Theorem 3.23., and p. 97, Remark 3.25.(ii). Consequently, denoting the minimal value of $(\mathrm{P})$ by $m$, we have

$$
m=\lim _{N^{\prime} \rightarrow \infty} F\left(\Phi^{N^{\prime}}, W^{N^{\prime}}, I_{e}^{N^{\prime}}\right) \geqslant \liminf _{N^{\prime} \rightarrow \infty} F\left(\Phi^{N^{\prime}}, W^{N^{\prime}}, I_{e}{ }^{N^{\prime}}\right) \geqslant F\left(\hat{\Phi}, \hat{W}, \hat{I}_{e}\right) \geqslant m,
$$

and $\left(\hat{\Phi}, \hat{W}, \hat{I}_{e}\right)$ is a global minimizer of $(\mathrm{P})$.

Remarks. 1) The analysis of this subsection and, particularly, Theorem 3.3. remain valid if the RogersMcCulloch model in (3.6) is replaced by the FitzHugh-Nagumo or the linearized Aliev-Panfilov model since the convergence arguments from the proof of Proposition 3.2. hold for the latter as well.

2) The situation where the excitations $I_{e}$ can be applied on a subdomain $\Omega_{\text {con }} \subset \Omega$ only, can be treated in a completely analogous way.

\section{c) The system of adjoint equations.}

For the optimal control problem $(\mathrm{P})$, we introduce the formal Lagrange function

$$
\begin{aligned}
\mathcal{L}\left(\Phi, W, I_{e}, P_{1}, P_{2}, P_{3}, P_{4}\right)=F\left(\Phi, W, I_{e}\right)+\left\langle P_{1},\right. & \left.E_{1}\left(\Phi, W, I_{e}\right)\right\rangle \\
& +\left\langle P_{2}, E_{2}(\Phi, W)\right\rangle+\left\langle P_{3}, E_{3}(\Phi)\right\rangle+\left\langle P_{4}, E_{4}(W)\right\rangle
\end{aligned}
$$

with multipliers $P_{1} \in L^{4}\left[(0, T), W^{1,2}(\Omega)\right], P_{2} \in L^{2}\left[(0, T), L^{2}(\Omega)\right]$, and $P_{3}, P_{4} \in\left(L^{2}(\Omega)\right)^{*}$. Differentiating $\mathcal{L}$ at the point $\left(\hat{\Phi}, \hat{W}, \hat{I}_{e}\right)$ in a formal way with respect to the variables $\Phi$ and $W$, we find the adjoint equations

$$
\begin{gathered}
D_{\Phi} F\left(\hat{\Phi}, \hat{W}, \hat{I}_{e}\right)+\left\langle P_{1}, D_{\Phi} E_{1}\left(\hat{\Phi}, \hat{W}, \hat{I}_{e}\right)\right\rangle+\left\langle P_{2}, D_{\Phi} E_{2}(\hat{\Phi}, \hat{W})\right\rangle+\left\langle P_{3}, D_{\Phi} E_{3}(\hat{\Phi})\right\rangle=0 \\
\Longleftrightarrow \int_{0}^{T} \int_{\Omega}\left(-\frac{\partial P_{1}}{\partial t}+\frac{\partial I_{\text {ion }}}{\partial \varphi}(\hat{\Phi}, \hat{W}) P_{1}\right) \psi d x d t+\frac{\lambda}{1+\lambda} \int_{0}^{T} \int_{\Omega} \nabla \psi^{\mathrm{T}} M_{i} \nabla P_{1} d x d t \\
\quad=-\int_{0}^{T} \int_{\Omega}\left(\frac{\partial r}{\partial \varphi}(\hat{\Phi}, \hat{W})+\frac{\partial G}{\partial \varphi}(\hat{\Phi}, \hat{W}) P_{2}\right) \psi d x d t-\int_{\Omega} P_{3} \psi(x, 0) d x \\
\forall \psi \in L^{2}\left[(0, T), W^{1,2}(\Omega)\right], P_{1}(x, T) \equiv 0 ;
\end{gathered}
$$

15) Cf. [Bourgault/Coudière/Pierre 09] , pp. 476 - 478, Subsection 5.4.3. 


$$
\begin{gathered}
D_{W} F\left(\hat{\Phi}, \hat{W}, \hat{I}_{e}\right)+\left\langle P_{1}, D_{W} E_{1}\left(\hat{\Phi}, \hat{W}, \hat{I}_{e}\right)\right\rangle+\left\langle P_{2}, D_{W} E_{2}(\hat{\Phi}, \hat{W})\right\rangle+\left\langle P_{4}, D_{W} E_{4}(\hat{W})\right\rangle=0 \\
\Longleftrightarrow \int_{0}^{T} \int_{\Omega}\left(-\frac{\partial P_{2}}{\partial t}+\frac{\partial G}{\partial w}(\hat{\Phi}, \hat{W}) P_{2}\right) \psi d x d t \\
=-\int_{0}^{T} \int_{\Omega}\left(\frac{\partial r}{\partial w}(\hat{\Phi}, \hat{W})+\frac{\partial I_{i o n}}{\partial w}(\hat{\Phi}, \hat{W}) P_{1}\right) \psi d x d t-\int_{\Omega} P_{4} \psi(x, 0) d x \\
\forall \psi \in L^{2}\left[(0, T), L^{2}(\Omega)\right], P_{2}(x, T) \equiv 0 .
\end{gathered}
$$

In Theorem 3.5. below, we will prove directly that this system is part of the necessary optimality conditions for $(\mathrm{P})$. The adjoint system consists of a parabolic PDE in its weak formulation, which is coupled with a linear ODE. Assuming that $P_{3}$ and $P_{4}$ may be set to zero, the corresponding strong formulation of the adjoint system reads as follows:

$$
\begin{gathered}
-\frac{\partial P_{1}}{\partial t}-\nabla \cdot\left(\frac{\lambda}{1+\lambda} M_{i} \nabla P_{1}\right)+\frac{\partial I_{i o n}}{\partial \varphi}(\hat{\Phi}, \hat{W}) P_{1}=-\frac{\partial G}{\partial \varphi}(\hat{\Phi}, \hat{W}) P_{2}-\frac{\partial r}{\partial \varphi}(\hat{\Phi}, \hat{W})(\forall)(x, t) \in \Omega_{T} ; \\
\mathfrak{n}^{\mathrm{T}} M_{i} \nabla P_{1}=0 \forall(x, t) \in \partial \Omega \times[0, T] ; \quad P_{1}(x, T)=0(\forall) x \in \Omega ; \\
-\frac{\partial P_{2}}{\partial t}+\frac{\partial G}{\partial w}(\hat{\Phi}, \hat{W}) P_{2}=-\frac{\partial I_{i o n}}{\partial w}(\hat{\Phi}, \hat{W}) P_{1}-\frac{\partial r}{\partial w}(\hat{\Phi}, \hat{W})(\forall)(x, t) \in \Omega_{T} ; \\
P_{2}(x, T)=0(\forall) x \in \Omega .
\end{gathered}
$$

\section{d) First-order necessary optimality conditions.}

We search for weak local minimizers according to the following definition:

Definition 3.4. (Weak local minimizer) A triple $\left(\hat{\Phi}, \hat{W}, \hat{I}_{e}\right)$, which is feasible in $(\mathrm{P})$, is called a weak local minimizer of $(\mathrm{P})$ iff there exists a number $\varepsilon>0$ such that for all admissible $\left(\Phi, W, I_{e}\right)$ the conditions

$$
\|\Phi-\hat{\Phi}\|_{\mathrm{X}_{1}} \leqslant \varepsilon,\|W-\hat{W}\|_{\mathrm{X}_{2}} \leqslant \varepsilon,\left\|I_{e}-\hat{I}_{e}\right\|_{\mathrm{X}_{3}} \leqslant \varepsilon
$$

imply the relation $F\left(\hat{\Phi}, \hat{W}, \hat{I}_{e}\right) \leqslant F\left(\Phi, W, I_{e}\right)$.

The existence of at least one weak local minimizer for $(\mathrm{P})$ is confirmed by Theorem 3.3. Treating $(\mathrm{P})$ as a "weakly singular problem", we may follow the approach outlined in [ITO/KunisCH 08], p. 17 f. and pp. 129 ff., ${ }^{16)}$ and prove the necessary optimality conditions without recourse to the regularity conditions of Kurcyusz-Zowe or Ioffe-Tichomirow. ${ }^{17)}$ Instead, the existence of a solution for the adjoint system will be ensured by a parabolic existence theorem (Theorem 3.10. below), the assumptions of which, consequently, must be carried over.

Theorem 3.5. (First-order necessary optimality conditions for the control problem (P)) We study the problem $(\mathrm{P})$ under the following assumptions:

a) $\Omega \subset \mathbb{R}^{3}$ is a bounded Lipschitz domain with piecewise $C^{1}$-boundary.

b) $M_{i}: \operatorname{cl}(\Omega) \rightarrow \mathbb{R}^{3 \times 3}$ is a symmetric, positive definite matrix function with $W^{1, \infty}(\Omega)$-coefficients, which obeys the uniform ellipticity condition (2.14).

16) Although the problem (P) does not fit all assumptions of [ Iто/KUNISCH 08] , p. 18, Theorem 1.17., the proof scheme of the theorem can be carried over.

17) Cf. [Ioffe/Tichomirow 79], p. 74, Assumption c), and [Iто/Kunisch 08], p. 5, Definition 1.5. 
c) The integrand $r(x, t, \varphi, w): \Omega \times[0, T] \times \mathbb{R} \times \mathbb{R}$ is bounded from below, measurable with respect to $x$ and $t$ and continuously differentiable and convex with respect to $\varphi$ and $w$.

The remaining assumptions from Subsection 3.a) are carried over.

If $\left(\hat{\Phi}, \hat{W}, \hat{I}_{e}\right) \in L^{2}\left[(0, T), W^{1,2}(\Omega)\right] \times L^{2}\left[(0, T), L^{2}(\Omega)\right] \times L^{\infty}\left[(0, T), L^{2}(\Omega)\right]$ is a weak local minimizer of $(\mathrm{P})$ with

$$
\frac{\partial r}{\partial \varphi}(\hat{\Phi}, \hat{W}), \frac{\partial r}{\partial w}(\hat{\Phi}, \hat{W}) \in L^{2}\left(\Omega_{T}\right)
$$

then there exist multipliers $P_{1} \in L^{2}\left[(0, T), W^{2,2}(\Omega)\right] \cap W^{1,2}\left[(0, T), L^{2}(\Omega)\right]$ and $P_{2} \in C^{1}[(0, T)$, $\left.L^{2}(\Omega)\right] \cap C^{0}\left[[0, T], L^{2}(\Omega)\right]$, satisfying together with $\left(\hat{\Phi}, \hat{W}, \hat{I}_{e}\right)$ the optimality condition

$$
\int_{0}^{T} \int_{\Omega}\left(\mu \hat{I}_{e}(x, t)+\frac{1}{1+\lambda} P_{1}(x, t)\right)\left(I_{e}(x, t)-\hat{I}_{e}(x, t)\right) d x d t \geqslant 0 \quad \forall I_{e} \in \mathcal{C}
$$

as well as the adjoint equations $(3.17)$ - (3.20). The functions $P_{1}$ and $P_{2}$ solve the adjoint system in the weak sense.

Note that, by the Aubin-Dubinskij lemma (Theorem 4.6.), the multiplier $P_{1}$ belongs even to the space $L^{4}\left[(0, T), W^{1,2}(\Omega)\right]$ as required in $(3.12)$.

Corollary 3.6. (Pointwise formulation of the optimality condition) The optimality condition (3.23) from Theorem 3.5. implies the following Pontryagin minimum condition, which holds a. e. pointwise:

$$
\left(\mu(1+\lambda) \hat{I}_{e}(x, t)+P_{1}(x, t)\right) \hat{I}_{e}(x, t)=\operatorname{Min}_{-R \leqslant \eta \leqslant R}\left(\mu(1+\lambda) \hat{I}_{e}(x, t)+P_{1}(x, t)\right) \eta \quad(\forall)(x, t) \in \Omega_{T} .
$$

Consequently, we have

$$
\hat{I}_{e}(x, t)=\left\{\begin{array}{c|l}
-R & Q(x, t)>R ; \\
-Q(x, t) & Q(x, t) \in[-R, R] ; \quad \text { where } Q(x, t)=\frac{1}{\mu(1+\lambda)} P_{1}(x, t) . \\
R & Q(x, t)<-R
\end{array}\right.
$$

We may conclude that an optimal control, which never becomes active, admits the same regularity as the adjoint variable $P_{1}$.

Corollary 3.7. (Higher regularity of weak local minimizers) Under the assumptions of Theorem 3.5., consider a weak local minimizer $\left(\hat{\Phi}, \hat{W}, \hat{I}_{e}\right)$ of $(\mathrm{P})$ with $\operatorname{ess} \sup _{(x, t) \in \Omega_{T}}\left|\hat{I}_{e}(x, t)\right|<R$. Then $\hat{I}_{e}$ belongs to the space $W^{1,2}\left[(0, T), L^{2}(\Omega)\right] \cap L^{2}\left[(0, T), W^{2,2}(\Omega)\right]$.

In a subsequent publication, we will prove that this corollary implies an improvement of the regularity of $\hat{\Phi}$ and $\hat{W}$ as well.

Remark. Theorem 3.5. as well as Corollaries 3.6. and 3.7. remain valid if the Rogers-McCulloch model in (3.6) is replaced by the FitzHugh-Nagumo model. If the linearized Aliev-Panfilov model is considered, the theorems hold still if (3.22) is replaced by

$$
\frac{\partial r}{\partial \varphi}(\hat{\Phi}, \hat{W}) \in L^{2}\left(\Omega_{T}\right) ; \quad \frac{\partial r}{\partial w}(\hat{\Phi}, \hat{W}) \in L^{2}\left[(0, T), L^{3}(\Omega)\right]
$$




\section{e) Proof of the necessary optimality conditions.}

As mentioned in the introduction, the proof of Theorem 3.5. requires a stability estimate for the weak solutions of the primal equations as well as an immediate existence result for the system of the adjoint equations. We start with the statement of the stability estimate:

Theorem 3.8. (Stability estimate for weak solutions of the primal equations) Assume that the data within (2.1) - (2.3) obey the assumptions of Theorem 2.2., and specify within the monodomain system any of the models from Subsection 2.b). If two weak solutions $\left(\Phi^{\prime}, W^{\prime}\right),\left(\Phi^{\prime \prime}, W^{\prime \prime}\right) \in\left(C^{0}[[0, T]\right.$, $\left.\left.L^{2}(\Omega)\right] \cap L^{2}\left[(0, T), W^{1,2}(\Omega)\right] \cap L^{4}\left(\Omega_{T}\right)\right) \times C^{0}\left[[0, T], L^{2}(\Omega)\right]$ of $(2.1)-(2.3)$ correspond with initial values $\Phi_{0}^{\prime}=\Phi_{0}^{\prime \prime}=\Phi_{0} \in L^{2}(\Omega), W_{0}^{\prime}=W_{0}^{\prime \prime}=W_{0} \in L^{4}(\Omega)$ and inhomogeneities $I_{i}{ }^{\prime}, I_{e}{ }^{\prime}, I_{i}{ }^{\prime \prime}$ and $I_{e}{ }^{\prime \prime} \in$ $L^{\infty}\left[(0, T),\left(W^{1,2}(\Omega)\right)^{*}\right]$, whose norms are bounded by $R>0$, then the following estimates hold:

$$
\begin{gathered}
\left\|\Phi^{\prime}-\Phi^{\prime \prime}\right\|_{C^{0}\left[[0, T], L^{2}(\Omega)\right]}^{2}+\left\|\Phi^{\prime}-\Phi^{\prime \prime}\right\|_{L^{2}\left[(0, T), W^{1,2}(\Omega)\right]}^{2}+\left\|W^{\prime}-W^{\prime \prime}\right\|_{W^{1,2}\left[(0, T), L^{2}(\Omega)\right]}^{2} \\
\leqslant C\left(\left\|I_{i}{ }^{\prime}-I_{i}{ }^{\prime \prime}\right\|_{L^{\infty}\left[(0, T),\left(W^{1,2}(\Omega)\right)^{*}\right]}^{2}+\left\|I_{e}{ }^{\prime}-I_{e}{ }^{\prime \prime}\right\|_{\left.L^{\infty}\left[(0, T),\left(W^{1,2}(\Omega)\right)^{*}\right]\right) .}^{2} .\right. \\
\left\|\Phi^{\prime}-\Phi^{\prime \prime}\right\|_{W^{1,4 / 3}\left[(0, T),\left(W^{1,2}(\Omega)\right)^{*}\right]} \leqslant C \cdot \operatorname{Max}\left(\left\|I_{i}{ }^{\prime}-I_{i}{ }^{\prime \prime}\right\|_{L^{2}\left[(0, T),\left(W^{1,2}(\Omega)\right)^{*}\right]},\right. \\
\left\|I_{e}{ }^{\prime}-I_{e}{ }^{\prime \prime}\right\|_{L^{2}\left[(0, T),\left(W^{1,2}(\Omega)\right)^{*}\right]},\left\|I_{i}{ }^{\prime}-I_{i}{ }^{\prime \prime}\right\|_{L^{2}\left[(0, T),\left(W^{1,2}(\Omega)\right)^{*}\right]}^{2},\left\|I_{e}{ }^{\prime}-I_{e}{ }^{\prime \prime}\right\|_{\left.L^{2}\left[(0, T),\left(W^{1,2}(\Omega)\right)^{*}\right]\right) .}^{2}
\end{gathered}
$$

The proof of Theorem 3.8. is postponed to Section 5 .

Theorem 3.9. (Existence of weak solutions for the adjoint system) Under the assumptions of Theorem 3.5., let $\left(\hat{\Phi}, \hat{W}, \hat{I}_{e}\right)$ be a feasible solution of $(\mathrm{P})$, satisfying

$$
\frac{\partial r}{\partial \varphi}(\hat{\Phi}, \hat{W}), \frac{\partial r}{\partial w}(\hat{\Phi}, \hat{W}) \in L^{2}\left(\Omega_{T}\right) .
$$

Then the adjoint system $(3.17)-(3.20)$ related to $\left(\hat{\Phi}, \hat{W}, \hat{I}_{e}\right)$ admits a weak solution $\left(P_{1}, P_{2}\right)$ with

$$
\begin{aligned}
& P_{1} \in L^{2}\left[(0, T), W^{2,2}(\Omega)\right] \cap W^{1,2}\left[(0, T), L^{2}(\Omega)\right] ; \\
& P_{2} \in C^{1}\left[(0, T), L^{2}(\Omega)\right] \cap C^{0}\left[[0, T], L^{2}(\Omega)\right] .
\end{aligned}
$$

Proof. The proof of Theorem 3.9. is based on the following parabolic existence theorem:

Theorem 3.10. (Existence theorem for the adjoint parabolic equation) ${ }^{18)}$ Consider the parabolic initial-boundary value problem

$$
\begin{aligned}
& \frac{\partial P}{\partial t}(x, t)-\sum_{i, j} \nabla \cdot\left(a_{i, j}(x, t) \nabla P(x, t)\right)+a_{0}(x, t) P(x, t)=f(x, t) \quad(\forall)(x, t) \in \Omega \times(0, T) ; \\
& \sum_{i, j} \mathfrak{n}_{i}(x) a_{i, j}(x, t) \frac{\partial P}{\partial x_{j}}(x, t)=g(x, t) \quad \forall(x, t) \in \partial \Omega \times(0, T) ; \quad P(x, 0)=P_{0}(x) \quad(\forall) x \in \Omega
\end{aligned}
$$

and assume the following about the data of (3.32) - (3.33):

a) $\Omega \subset \mathbb{R}^{n}, n \geqslant 3$, is a bounded Lipschitz domain with piecewise $C^{1}$-boundary.

b) The matrix function $a_{i j}: \operatorname{cl}(\Omega) \times[0, T] \rightarrow \mathbb{R}^{n \times n}$ with weakly differentiable entries is symmetric and satisfies a uniform ellipticity condition. It holds that

$$
\begin{aligned}
& \frac{\partial a_{i j}}{\partial x_{k}} \in L^{r_{1}}\left[(0, T), L^{q_{1}}(\Omega)\right] \text { with } \frac{1}{r_{1}}+\frac{n}{2 q_{1}}=\frac{1}{2}, 2<r_{1}<\infty ; \\
& \frac{\partial a_{i j}}{\partial t} \in L^{1}\left[(0, T), L^{\infty}(\Omega)\right] .
\end{aligned}
$$

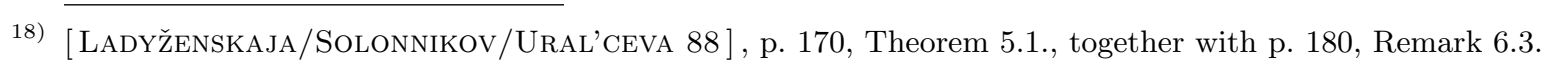


c) $a_{0} \in L^{2}\left[(0, T), L^{n}(\Omega)\right] \cap L^{r_{2}}\left[(0, T), L^{q_{2}}(\Omega)\right]$ with $\frac{1}{r_{2}}+\frac{n}{2 q_{2}}=1,1 \leqslant r_{2}<\infty, 2<q_{2} \leqslant \infty$.

d) $f \in L^{2}\left(\Omega_{T}\right)$.

e) $g \in L^{r_{3}}\left[(0, T), L^{q_{3}}(\partial \Omega)\right]$ with $\frac{1}{r_{3}}+\frac{n-1}{2 q_{3}}=\frac{1}{2}+\frac{n}{4}, 1 \leqslant r_{3} \leqslant 2, \frac{2 n-1}{n} \leqslant q_{3} \leqslant \frac{2 n-2}{n-2}$.

f) $P_{0} \in W^{2,2}(\Omega)$.

Then problem $(3.32)$ - (3.33) admits a weak solution $P \in L^{2}\left[(0, T), W^{2,2}(\Omega)\right] \cap W^{1,2}\left[(0, T), L^{2}(\Omega)\right]$, which satisfies the estimate

$$
\begin{aligned}
\|P\|_{L^{2}\left[(0, T), W^{2,2}(\Omega)\right]}+\|P\|_{W^{1,2}\left[(0, T), L^{2}(\Omega)\right]} & (3.36) \\
& \left.\leqslant C\left(\|f\|_{L^{2}\left[(0, T), L^{2}(\Omega)\right]}+\|g\|_{L^{r_{3}\left[(0, T), L^{q_{3}(\partial \Omega)}\right]}}\right)\right)
\end{aligned}
$$

with a constant $C>0$ depending on $q_{1}, q_{2}, q_{3}, r_{1}, r_{2}, r_{3}, T$ and $\Omega$.

Since the Rogers-McCulloch model has been specified within (P), the adjoint equations (3.17) and (3.19) read as follows:

$$
\begin{aligned}
& -\frac{\partial P_{1}}{\partial t}-\nabla \cdot\left(\frac{\lambda}{1+\lambda} M_{i} \nabla P_{1}\right)+\left(3 b(\hat{\Phi})^{2}-2(a+1) b \hat{\Phi}+a b+\hat{W}\right) P_{1}=\varepsilon \kappa P_{2}-\frac{\partial r}{\partial \varphi}(\hat{\Phi}, \hat{W}) ; \\
& -\frac{\partial P_{2}}{\partial t}+\varepsilon P_{2}=-\hat{\Phi} P_{1}-\frac{\partial r}{\partial w}(\hat{\Phi}, \hat{W}) .
\end{aligned}
$$

- Step 1. Improved regularity of $\hat{\Phi}$ and $\hat{W}$ from $\hat{I}_{e} \in L^{\infty}\left[(0, T), L^{2}(\Omega)\right]$. Within the proof of Theorem 2.4., the following estimate is derived: ${ }^{19)}$

$$
\begin{gathered}
\|\hat{\Phi}(t)\|_{W^{1,2}(\Omega)}^{2} \leqslant C\left(1+\left\|\Phi_{0}\right\|_{L^{2}(\Omega)}^{2}+\left\|W_{0}\right\|_{L^{2}(\Omega)}^{2}+\int_{0}^{t}\left\|\hat{I}_{e}(\tau)\right\|_{\left(W^{1,2}(\Omega)\right.}^{2}\right)^{*} d \tau+\left\|\hat{I}_{e}(t)\right\|_{\left.\left(W^{1,2}(\Omega)\right)^{*}\right)}^{2} \\
\leqslant C\left(1+\left\|\Phi_{0}\right\|_{L^{2}(\Omega)}^{2}+\left\|W_{0}\right\|_{L^{2}(\Omega)}^{2}+\left\|\hat{I}_{e}\right\|_{L^{2}\left(\Omega_{T}\right)}^{2}+\left\|\hat{I}_{e}\right\|_{L^{\infty}\left[(0, T), L^{2}(\Omega)\right]}^{2}\right)
\end{gathered}
$$

for arbitrary $t \in[0, T]$. Consequently, $\hat{\Phi} \in L^{\infty}\left[(0, T), W^{1,2}(\Omega)\right]$. Note that

$$
\hat{W}(x, t)=W_{0}(x) e^{-\varepsilon t}+\varepsilon \kappa \int_{0}^{t} \hat{\Phi}(x, \tau) e^{\varepsilon(\tau-t)} d \tau
$$

belongs to $C^{0}\left[[0, T], L^{4}(\Omega)\right]$ together with $\hat{\Phi}(\tau), W_{0} \in L^{4}(\Omega)$.

- Step 2. For any $\widetilde{P}_{1} \in L^{4}\left(\Omega_{T}\right)$, the terminal problem for the adjoint ODE admits a unique (weak or strong) solution $P_{2} \in C^{1}\left[(0, T), L^{2}(\Omega)\right] \cap C^{0}\left[[0, T], L^{2}(\Omega)\right]$. It is obvious that the problem

$$
-\frac{\partial P_{2}}{\partial t}+\varepsilon P_{2}=-\hat{\Phi} \widetilde{P}_{1}-\frac{\partial r}{\partial w}(\hat{\Phi}, \hat{W}) \quad(\forall)(x, t) \in \Omega_{T}, P_{2}(x, T) \equiv 0
$$

admits the unique solution

$$
P_{2}(x, t)=-\int_{t}^{T}\left(\hat{\Phi} \widetilde{P}_{1}+\frac{\partial r}{\partial w}(\hat{\Phi}, \hat{W})\right) e^{\varepsilon(t-\tau)} d \tau
$$

which is continuous in time on $[0, T]$ and even differentiable in time on $(0, T)$. In order to confirm the integrability with respect to $x$, we estimate

$$
\int_{\Omega}\left(\hat{\Phi}(t) \widetilde{P}_{1}(t)\right)^{2} d x \leqslant\left(\int_{\Omega}|\hat{\Phi}(t)|^{4} d x\right)^{1 / 2}\left(\int_{\Omega}\left|\widetilde{P}_{1}(t)\right|^{4} d x\right)^{1 / 2}
$$

19) From [Bourgault/Coudière/Pierre 09], p. 474, (67), using the Gronwall inequality and inserting $I_{i}=\mathfrak{o}$. 
where the right-hand side is finite due to the continuous imbedding $\hat{\Phi}(t) \in W^{1,2}(\Omega) \hookrightarrow L^{4}(\Omega)$. Consequently, $P_{2}$ belongs to the space $C^{1}\left[(0, T), L^{2}(\Omega)\right] \cap C^{0}\left[[0, T], L^{2}(\Omega)\right]$.

- Step 3. For any $\widetilde{P}_{2} \in L^{2}\left(\Omega_{T}\right)$, the terminal-boundary value problem for the parabolic adjoint equation admits a unique weak solution $P_{1} \in L^{2}\left[(0, T), W^{2,2}(\Omega)\right] \cap W^{1,2}\left[(0, T), L^{2}(\Omega)\right]$. In order to confirm this claim, we must check whether the assumptions of Theorem 3.10. are satisfied. Note first that the assertions of the theorem will not be afflicted by the fact that time runs backwards from $T$. Assumption a) is identical with Assumption a) of Theorem 3.5.; in order to confirm b) note that the Lipschitz entries of $M_{i}$ are time-independent, thus (3.34) is satisfied e. g. with $r_{1}=4, q_{1}=6$, and (3.35) holds true as well. By Step $1, \hat{\Phi}$ belongs particularly to $L^{\infty}\left[(0, T), L^{6}(\Omega)\right]$, which implies $(\hat{\Phi})^{2} \in L^{\infty}\left[(0, T), L^{3}(\Omega)\right]$. Since $\hat{W} \in C^{0}\left[[0, T], L^{4}(\Omega)\right]$ as well, we find that

$$
a_{0}=\left(3 b(\hat{\Phi})^{2}-2(a+1) b \hat{\Phi}+a b+\hat{W}\right) \in L^{\infty}\left[[0, T], L^{3}(\Omega)\right],
$$

and Assumption c) is satisfied with $r_{2}=2, q_{2}=3$. By (3.29), Assumption d) holds true as well, and the functions $g(x, t) \equiv 0$ and $P_{0} \equiv 0$ fulfill e) with e. g. $r_{3}=4 / 3, q_{3}=2$, and f) as well. Consequently, the terminal-boundary problem (3.17) - (3.18) admits a weak solution $P_{1}$ with the claimed regularity.

- Step 4. For two functions $P_{1}^{\prime}, P_{1}^{\prime \prime} \in L^{4}\left(\Omega_{T}\right)$, the corresponding solutions of the terminal problem for the adjoint ODE satisfy

$$
\left\|P_{2}^{\prime}(t)-P_{2}^{\prime \prime}(t)\right\|_{L^{2}(\Omega)}^{2} \leqslant C \cdot \int_{t}^{T}\left\|P_{1}^{\prime}(\tau)-P_{1}^{\prime \prime}(\tau)\right\|_{L^{4}(\Omega)}^{2} d \tau .
$$

The solutions of the ODE have been calculated in Step 2. Consequently, applying Jensen's integral inequality and Hölder's inequality, we find

$$
\begin{aligned}
& P_{2}^{\prime}(x, t)-P_{2}^{\prime \prime}(x, t)= \int_{t}^{T} \hat{\Phi}\left(P_{1}^{\prime \prime}-P_{1}^{\prime}\right) e^{\varepsilon(t-\tau)} d \tau \Longrightarrow\left|P_{2}^{\prime}-P_{2}^{\prime \prime}\right| \leqslant \int_{t}^{T}|\hat{\Phi}| \cdot\left|P_{1}^{\prime}-P_{1}^{\prime \prime}\right| d \tau \Longrightarrow \\
&\left|P_{2}^{\prime}-P_{2}^{\prime \prime}\right|^{2} \leqslant(T-t)^{2}\left(\frac{1}{T-t} \int_{t}^{T}|\hat{\Phi}| \cdot\left|P_{1}^{\prime}-P_{1}^{\prime \prime}\right| d \tau\right)^{2} \leqslant T \int_{t}^{T}|\hat{\Phi}|^{2} \cdot\left|P_{1}^{\prime}-P_{1}^{\prime \prime}\right|^{2} d \tau \Longrightarrow \\
& \int_{\Omega}\left|P_{2}^{\prime}-P_{2}^{\prime \prime}\right|^{2} d x \leqslant C \int_{t}^{T} \int_{\Omega}|\hat{\Phi}|^{2} \cdot\left|P_{1}^{\prime}-P_{1}^{\prime \prime}\right|^{2} d x d \tau \leqslant C \int_{t}^{T}\left(\int_{\Omega}|\hat{\Phi}|^{4} d x\right)^{1 / 2} \\
& \cdot\left(\int_{\Omega}\left|P_{1}^{\prime}-P_{1}^{\prime \prime}\right|^{4} d x\right)^{1 / 2} d \tau=C \int_{t}^{T}\|\hat{\Phi}(\tau)\|_{L^{4}(\Omega)}^{2} \cdot\left\|P_{1}^{\prime}(\tau)-P_{1}^{\prime \prime}(\tau)\right\|_{L^{4}(\Omega)}^{2} d \tau .
\end{aligned}
$$

From Step 1, we know that $\hat{\Phi} \in L^{\infty}\left[(0, T), W^{1,2}(\Omega)\right] \hookrightarrow L^{\infty}\left[(0, T), L^{4}(\Omega)\right]$. Consequently, the norms $\|\hat{\Phi}(\tau)\|_{L^{4}(\Omega)}^{2}$ are uniformly bounded with respect to $\tau$, and we arrive at the claimed inequality.

- Step 5. For two functions $P_{2}^{\prime}, P_{2}^{\prime \prime} \in L^{2}\left(\Omega_{T}\right)$, the corresponding solutions of the terminal-boundary value problem for the parabolic adjoint equation satisfy

$$
\left\|P_{1}^{\prime}(t)-P_{1}^{\prime \prime}(t)\right\|_{L^{4}(\Omega)}^{2} \leqslant C \cdot \int_{t}^{T}\left\|P_{2}^{\prime}(\tau)-P_{2}^{\prime \prime}(\tau)\right\|_{L^{2}(\Omega)}^{2} d \tau .
$$

In its strong formulation, the difference of the linear parabolic equations determining $P_{1}^{\prime}$ and $P_{1}^{\prime \prime}$ reads as

$$
\begin{aligned}
&-\frac{\partial}{\partial t}\left(P_{1}^{\prime}-P_{1}^{\prime \prime}\right)-\nabla \cdot\left(\frac{\lambda}{\lambda+1} M_{i} \nabla\left(P_{1}^{\prime}-P_{1}^{\prime \prime}\right)\right) \\
&+\left(3 b(\hat{\Phi})^{2}-2(a+1) b \hat{\Phi}+a b+\hat{W}\right)\left(P_{1}^{\prime}-P_{1}^{\prime \prime}\right)=\varepsilon \kappa\left(P_{2}^{\prime}-P_{2}^{\prime \prime}\right) ; \\
& \mathfrak{n}^{\mathrm{T}} M_{i} \nabla\left(P_{1}^{\prime}-P_{1}^{\prime \prime}\right)=0 ; \quad\left(P_{1}^{\prime}-P_{1}^{\prime \prime}\right)(x, T) \equiv 0,
\end{aligned}
$$


and from Theorem 3.10., we get the a-priori estimate

$$
\begin{aligned}
& \left.C\left(\int_{t}^{T}\left\|P_{2}^{\prime}-P_{2}^{\prime \prime}\right\|_{L^{2}(\Omega)}^{2} d \tau\right)^{1 / 2}=C\left\|P_{2}^{\prime}-P_{2}^{\prime \prime}\right\|_{L^{2}\left[(t, T), L^{2}(\Omega)\right.}\right] \\
& \geqslant C\left(\left\|P_{1}^{\prime}-P_{1}^{\prime \prime}\right\|_{L^{2}\left[(t, T), W^{2,2}(\Omega)\right.}+\left\|P_{1}^{\prime}-P_{1}^{\prime \prime}\right\|_{W^{1,2}\left[(t, T), L^{2}(\Omega)\right]}\right) \\
& \geqslant\left\|P_{1}^{\prime}-P_{1}^{\prime \prime}\right\|_{C^{0}\left[[t, T], W^{1,2}(\Omega)\right]} \geqslant\left\|P_{1}^{\prime}(t)-P_{1}^{\prime \prime}(t)\right\|_{L^{4}(\Omega)},
\end{aligned}
$$

cf. [Evans 98], p. 287, Theorem 3.

- Step 6. Application of Banach's fixed point theorem. ${ }^{20)}$ We consider the operator

$$
\mathcal{I}:\left(L^{2}\left[(0, T), L^{4}(\Omega)\right] \times L^{2}\left(\Omega_{T}\right)\right) \rightarrow\left(L^{2}\left[(0, T), L^{4}(\Omega)\right] \times L^{2}\left(\Omega_{T}\right)\right),
$$

which assigns to a given pair $\left(P_{1}, P_{2}\right)$ the new pair $\left(\mathcal{I} P_{1}, \mathcal{I} P_{2}\right)$ arising from the solution $\mathcal{I} P_{2}$ of the adjoint ODE after insertion of $P_{1}$ and the solution of the adjoint parabolic problem after insertion of $\mathcal{I} P_{2}$. In order to prove the contractivity of this operator, we start with two pairs $\left(P_{1}^{\prime}, P_{2}^{\prime}\right),\left(P_{1}^{\prime \prime}, P_{2}^{\prime \prime}\right) \in L^{2}[(0, T)$, $\left.L^{4}(\Omega)\right] \times L^{2}\left(\Omega_{T}\right)$. By (3.46) and (3.51), it holds that

$$
\begin{aligned}
& \left\|\mathcal{I} P_{1}^{\prime}(t)-\mathcal{I} P_{1}^{\prime \prime}(t)\right\|_{L^{4}(\Omega)}^{2} \leqslant C \int_{t}^{T}\left\|\mathcal{I} P_{2}^{\prime}(\tau)-\mathcal{I} P_{2}^{\prime \prime}(\tau)\right\|_{L^{2}(\Omega)}^{2} d \tau \\
& \quad \leqslant C \int_{t}^{T} \int_{\tau}^{T}\left\|P_{1}^{\prime}(\vartheta)-P_{1}^{\prime \prime}(\vartheta)\right\|_{L^{4}(\Omega)}^{2} d \vartheta d \tau \leqslant C T \cdot \int_{t}^{T}\left\|P_{1}^{\prime}(\vartheta)-P_{1}^{\prime \prime}(\vartheta)\right\|_{L^{4}(\Omega)}^{2} d \vartheta .
\end{aligned}
$$

Defining the functions

$$
f(t)=\left\|\mathcal{I} P_{1}^{\prime}(t)-\mathcal{I} P_{1}^{\prime \prime}(t)\right\|_{L^{4}(\Omega)}^{2} \text { and } \tilde{f}(t)=\left\|P_{1}^{\prime}(t)-P_{1}^{\prime \prime}(t)\right\|_{L^{4}(\Omega)}^{2},
$$

this inequality reads as

$$
\begin{aligned}
0 & \leqslant f(t) \leqslant C \int_{t}^{T} \tilde{f}(\vartheta) d \vartheta \Longrightarrow \int_{0}^{T} e^{\lambda_{1} t} f(t) d t \leqslant C \cdot \int_{0}^{T} e^{\lambda_{1} t}\left(\int_{t}^{T} \tilde{f}(\vartheta) d \vartheta\right) d t \\
& =C\left[\frac{1}{\lambda_{1}} e^{\lambda_{1} t} \cdot \int_{t}^{T} \tilde{f}(\vartheta) d \vartheta\right]_{0}^{T}+C \int_{0}^{T} \frac{1}{\lambda_{1}} e^{\lambda_{1} t} \widetilde{f}(t) d t \\
& =\frac{C}{\lambda_{1}}\left(\int_{0}^{T} e^{\lambda_{1} t} \tilde{f}(t) d t-\int_{0}^{T} \tilde{f}(\vartheta) d \vartheta\right) \leqslant \frac{C}{\lambda_{1}} \int_{0}^{T} e^{\lambda_{1} t} \widetilde{f}(t) d t
\end{aligned}
$$

since the second member within the brackets is positive. Consequently, the operator $\mathcal{I}$ is with respect to its first component contractive on the space $L^{2}\left[(0, T), L^{4}(\Omega)\right]$ if this space is equipped with the equivalent norm

$$
\left\|P_{1}\right\|=\left(\int_{0}^{T} e^{\lambda_{1} t}\left\|P_{1}(t)\right\|_{L^{4}(\Omega)}^{2} d t\right)^{1 / 2}
$$

with sufficiently large $\lambda_{1}>C$. Analogously, we may estimate

$$
\begin{aligned}
& \left\|\mathcal{I} P_{2}^{\prime}(t)-\mathcal{I} P_{2}^{\prime \prime}(t)\right\|_{L^{2}(\Omega)}^{2} \leqslant C \cdot \int_{t}^{T}\left\|P_{1}^{\prime}(\tau)-P_{1}^{\prime \prime}(\tau)\right\|_{L^{4}(\Omega)}^{2} d \tau \\
& \quad \leqslant C \cdot \int_{t}^{T} \int_{\tau}^{T}\left\|P_{2}^{\prime}(\vartheta)-P_{2}^{\prime \prime}(\vartheta)\right\|_{L^{2}(\Omega)}^{2} d \vartheta d \tau \leqslant C T \cdot \int_{t}^{T}\left\|P_{2}^{\prime}(\vartheta)-P_{2}^{\prime \prime}(\vartheta)\right\|_{L^{2}(\Omega)}^{2} d \vartheta .
\end{aligned}
$$

20) Cf. [Veneroni 09], p. 866. 
With the abbreviations

$$
h(t)=\left\|\mathcal{I} P_{2}^{\prime}(t)-\mathcal{I} P_{2}^{\prime \prime}(t)\right\|_{L^{2}(\Omega)}^{2} \text { and } \tilde{h}(t)=\left\|P_{2}^{\prime}(t)-P_{2}^{\prime \prime}(t)\right\|_{L^{2}(\Omega)}^{2},
$$

the last inequality reads as

$$
\begin{aligned}
0 & \leqslant h(t) \leqslant C \int_{t}^{T} \widetilde{h}(\vartheta) d \vartheta \Longrightarrow \int_{0}^{T} e^{\lambda_{2} t} h(t) d t \leqslant C \cdot \int_{0}^{T} e^{\lambda_{2} t}\left(\int_{t}^{T} \widetilde{h}(\vartheta) d \vartheta\right) d t \\
& =C\left[\frac{1}{\lambda_{2}} e^{\lambda_{2} t} \cdot \int_{t}^{T} \widetilde{h}(\vartheta) d \vartheta\right]_{0}^{T}+C \int_{0}^{T} \frac{1}{\lambda_{2}} e^{\lambda_{2} t} \widetilde{h}(t) d t \\
& =\frac{C}{\lambda_{2}}\left(\int_{0}^{T} e^{\lambda_{2}} t \widetilde{h}(t) d t-\int_{0}^{T} \widetilde{h}(\vartheta) d \vartheta\right) \leqslant \frac{C}{\lambda_{2}} \int_{0}^{T} e^{\lambda_{2}} t \widetilde{h}(t) d t
\end{aligned}
$$

since the second member within the brackets is positive again. This implies the contractivity of the operator $\mathcal{I}$ with respect to its second component on the space $L^{2}\left(\Omega_{T}\right)$ if this space is equipped with the equivalent norm

$$
\left\|P_{2}\right\|=\left(\int_{0}^{T} e^{\lambda_{2} t}\left\|P_{2}(t)\right\|_{L^{2}(\Omega)}^{2} d t\right)^{1 / 2}
$$

with sufficiently large $\lambda_{2}>C$. Summing up, Banach's fixed point theorem yields the existence and uniqueness of a weak solution for the adjoint system, which admits the regularity guaranteed by Theorem 3.10. and Step 2 above.

Remarks. 1) If the Rogers-McCulloch model in (3.6) is replaced by the FitzHugh-Nagumo model, Theorem 3.9. remains valid. Then the adjoint equations (3.17) and (3.19) read as follows:

$$
\begin{aligned}
& -\frac{\partial P_{1}}{\partial t}-\nabla \cdot\left(\frac{\lambda}{\lambda+1} M_{i} \nabla P_{1}\right)+\left(3(\hat{\Phi})^{2}-2(a+1) \hat{\Phi}+a\right) P_{1}=\varepsilon \kappa P_{2}-\frac{\partial r}{\partial \varphi}(\hat{\Phi}, \hat{W}) ; \\
& -\frac{\partial P_{2}}{\partial t}+\varepsilon P_{2}=-P_{1}-\frac{\partial r}{\partial w}(\hat{\Phi}, \hat{W}),
\end{aligned}
$$

and the proof runs as above with minor alterations. In (3.45) and (3.52), the variable coefficient must be replaced by $a_{0}=\left(3(\hat{\Phi})^{2}-2(a+1) \hat{\Phi}+a\right)$, which belongs to $L^{2}\left[(0, T), L^{3}(\Omega)\right]$ as well. In Steps 2 and 4 , the right-hand side of the adjoint ODE simplifies accordingly.

2) In the case of the linearized Aliev-Panfilov model, Theorem 3.9. remains valid if the assumption (3.29) is replaced by (3.26). Here the adjoint equations (3.17) and (3.19) become

$$
\begin{aligned}
&-\frac{\partial P_{1}}{\partial t}-\nabla \cdot\left(\frac{\lambda}{\lambda+1} M_{i} \nabla P_{1}\right)+\left(3 b(\hat{\Phi})^{2}-2(a+1) b \hat{\Phi}+a b+\hat{W}\right) P_{1} \\
&=\varepsilon \kappa((a+1)-2 \hat{\Phi}) P_{2}-\frac{\partial r}{\partial \varphi}(\hat{\Phi}, \hat{W}) ;
\end{aligned}
$$

$-\frac{\partial P_{2}}{\partial t}+\varepsilon P_{2}=-\hat{\Phi} P_{1}-\frac{\partial r}{\partial w}(\hat{\Phi}, \hat{W})$.

In Step 2, noticing (3.26), we must work with $\widetilde{P}_{1} \in L^{6}\left(\Omega_{T}\right)$ in order to get $P_{2} \in C^{1}\left[(0, T), L^{3}(\Omega)\right] \cap$ $C^{0}\left[[0, T], L^{3}(\Omega)\right]$. In Step 3, Assumption d) of Theorem 3.10., namely $\left(\hat{\Phi} \widetilde{P}_{2}\right) \in L^{2}\left(\Omega_{T}\right)$, can be confirmed by inserting $\widetilde{P}_{2} \in L^{2}\left[(0, T), L^{3}(\Omega)\right]$, thus getting again $P_{1} \in L^{2}\left[[0, T], W^{2,2}(\Omega)\right] \cap W^{1,2}[[0, T]$, $\left.L^{2}(\Omega)\right] \hookrightarrow L^{6}\left(\Omega_{T}\right)$. Accordingly, (3.46) and (3.51) will be replaced by

$$
\begin{aligned}
& \left\|P_{2}^{\prime}(t)-P_{2}^{\prime \prime}(t)\right\|_{L^{3}(\Omega)}^{3} \leqslant C \cdot \int_{t}^{T}\left\|P_{1}^{\prime}(\tau)-P_{1}^{\prime \prime}(\tau)\right\|_{L^{6}(\Omega)}^{3} d \tau \quad \text { and } \\
& \left\|P_{1}^{\prime}(t)-P_{1}^{\prime \prime}(t)\right\|_{L^{6}(\Omega)}^{3} \leqslant C \cdot \int_{t}^{T}\left\|P_{2}^{\prime}(\tau)-P_{2}^{\prime \prime}(\tau)\right\|_{L^{3}(\Omega)}^{3} d \tau
\end{aligned}
$$


and the proof can be completed accordingly.

Proof of Theorem 3.5. • Step 1. Assume that $\left(\hat{\Phi}, \hat{W}, \hat{I}_{e}\right)$ is a weak local minimizer of $(\mathrm{P})$. If $I_{e} \in \mathcal{C}$ is an arbitrary feasible control with $\left\|I_{e}-\hat{I}_{e}\right\|_{L^{\infty}\left[(0, T), L^{2}(\Omega)\right.} \leqslant \varepsilon$ then, by Proposition 3.1., all controls

$$
I_{e}(s)=\hat{I}_{e}+s\left(I_{e}-\hat{I}_{e}\right), 0 \leqslant s \leqslant 1,
$$

belong to $\mathcal{C}$ as well. By Theorem 2.2., for every $I_{e}(s) \in L^{\infty}\left[(0, T), L^{2}(\Omega)\right]$, there exists a corresponding weak solution $(\Phi(s), W(s)) \in \mathrm{X}_{1} \times \mathrm{X}_{2}$ for the monodomain problem on $[0, T]$. Consequently, the triples $\left(\Phi(s), W(s), I_{e}(s)\right)$ are feasible in (P) for all $0 \leqslant s \leqslant 1$. On the other hand, from Theorem 3.8. it follows that every feasible triple within the neighborhood $\mathrm{K}(\hat{\Phi}, C \varepsilon) \times \mathrm{K}(\hat{W}, C \varepsilon) \times \mathrm{K}\left(\hat{I}_{e}, \varepsilon\right)$ can be generated in this way.

- Step 2. Lemma 3.11. For all $I_{e} \in \mathcal{C},\left\|I_{e}-\hat{I}_{e}\right\|_{L^{\infty}\left[(0, T), L^{2}(\Omega)\right.} \leqslant \varepsilon$ implies that

$$
\begin{aligned}
& \lim _{s \rightarrow 0+0} \frac{1}{s}\|\Phi(s)-\hat{\Phi}\|_{\mathrm{X}_{1}}^{2}=0 ; \quad \lim _{s \rightarrow 0+0} \frac{1}{s}\|\Phi(s)-\hat{\Phi}\|_{\widetilde{\mathrm{X}}_{1}}^{2}=0 ; \\
& \lim _{s \rightarrow 0+0} \frac{1}{s}\|W(s)-\hat{W}\|_{\mathrm{X}_{2}}^{2}=0 \text { and } \lim _{s \rightarrow 0+0} \frac{1}{s}\|W(s)-\hat{W}\|_{\widetilde{\mathrm{X}}_{2}}^{2}=0 .
\end{aligned}
$$

Proof. From Theorem 3.8., (3.27), we derive

$$
\begin{gathered}
\left.\|\Phi(s)-\hat{\Phi}\|_{\mathrm{X}_{1}}^{2}=\|\Phi(s)-\hat{\Phi}\|_{L^{2}}^{2}\left[(0, T), W^{1,2}(\Omega)\right] \leqslant C \cdot\left\|I_{e}(s)-\hat{I}_{e}\right\|_{L^{\infty}\left[(0, T),\left(W^{1,2}(\Omega)\right)^{*}\right]}^{2}\right] \\
\leqslant C \cdot\left\|I_{e}(s)-\hat{I}_{e}\right\|_{L^{\infty}}^{2}\left[(0, T), L^{2}(\Omega)\right]=C s^{2}\left\|I_{e}-\hat{I}_{e}\right\|_{L^{\infty}\left[(0, T), L^{2}(\Omega)\right]}^{2} \Longrightarrow \\
\lim _{s \rightarrow 0+0} \frac{1}{s}\|\Phi(s)-\hat{\Phi}\|_{\mathrm{X}_{1}}^{2} \leqslant \lim _{s \rightarrow 0+0} C s\left\|I_{e}-\hat{I}_{e}\right\|_{L^{\infty}\left[(0, T), L^{2}(\Omega)\right.}^{2}=0
\end{gathered}
$$

as well as

$$
\begin{gathered}
\|W(s)-\hat{W}\|_{\mathrm{X}_{2}}^{2}=\|W(s)-\hat{W}\|_{L^{2}\left[(0, T), L^{2}(\Omega)\right]}^{2} \leqslant C \cdot\left\|I_{e}(s)-\hat{I}_{e}\right\|_{L^{\infty}}^{2}\left[(0, T),\left(W^{1,2}(\Omega)\right)^{*}\right] \\
\left.\leqslant C \cdot\left\|I_{e}(s)-\hat{I}_{e}\right\|_{L^{\infty}}^{2}\left[(0, T), L^{2}(\Omega)\right]=C s^{2}\left\|I_{e}-\hat{I}_{e}\right\|_{L^{\infty}\left[(0, T), L^{2}(\Omega)\right.}^{2}\right] \Longrightarrow \\
\lim _{s \rightarrow 0+0} \frac{1}{s}\|W(s)-\hat{W}\|_{\mathrm{X}_{2}}^{2} \leqslant \lim _{s \rightarrow 0+0} C s\left\|I_{e}-\hat{I}_{e}\right\|_{L^{\infty}}^{2}\left[(0, T), L^{2}(\Omega)\right]=0 .
\end{gathered}
$$

The relation with $\|W(s)-\hat{W}\|_{\widetilde{\mathrm{x}}_{2}}^{2}$ can be confirmed analogously. Finally, (3.28) implies that

$$
\begin{aligned}
& \|\Phi(s)-\hat{\Phi}\|_{\widetilde{\mathrm{X}}_{1}}^{2}=\|\Phi(s)-\hat{\Phi}\|_{W^{1,4 / 3}\left[(0, T),\left(W^{1,2}(\Omega)\right)^{*}\right]}^{2} \\
& \leqslant C^{2} \cdot \operatorname{Max}\left(\left\|I_{e}(s)-\hat{I}_{e}\right\|_{L^{\infty}\left[(0, T),\left(W^{1,2}(\Omega)\right)^{*}\right],\left\|I_{e}(s)-\hat{I}_{e}\right\|_{L^{\infty}}^{4}\left[(0, T),\left(W^{1,2}(\Omega)\right)^{*}\right]}^{2}\right. \\
& \leqslant C \cdot \operatorname{Max}\left(s^{2}\left\|I_{e}-\hat{I}_{e}\right\|_{L^{\infty}\left[(0, T), L^{2}(\Omega)\right]}^{2} s^{4}\left\|I_{e}-\hat{I}_{e}\right\|_{L^{\infty}\left[(0, T), L^{2}(\Omega)\right]}^{4}\right) \Longrightarrow \\
& \lim _{s \rightarrow 0+0} \frac{1}{s}\|\Phi(s)-\hat{\Phi}\|_{\mathrm{X}_{1}}^{2} \\
& \quad \leqslant \lim _{s \rightarrow 0+0} C \cdot \operatorname{Max}\left(s\left\|I_{e}-\hat{I}_{e}\right\|_{L^{\infty}\left[(0, T), L^{2}(\Omega)\right]}^{2}, s^{3}\left\|I_{e}-\hat{I}_{e}\right\|_{L^{\infty}\left[(0, T), L^{2}(\Omega)\right]}^{4}\right)
\end{aligned}
$$

- Step 3. By Theorem 3.9., there exist solutions $P_{1} \in\left(L^{4 / 3}\left[(0, T),\left(W^{1,2}(\Omega)\right)^{*}\right]\right)^{*}=L^{4}[(0, T)$, $\left.W^{1,2}(\Omega)\right]$ and $P_{2} \in\left(L^{2}\left[(0, T),\left(L^{2}(\Omega)\right)^{*}\right]\right)^{*}=L^{2}\left(\Omega_{T}\right)$ for the adjoint system in relation to $\left(\hat{\Phi}, \hat{W}, \hat{I}_{e}\right)$. Using these functions, we get the following estimates: 
Lemma 3.12. With the notations of Subsection 3.a), it holds that

$\lim _{s \rightarrow 0+0} \frac{1}{s}\left\langle P_{1}, D_{\Phi} E_{1}\left(\hat{\Phi}, \hat{W}, \hat{I}_{e}\right)(\Phi(s)-\hat{\Phi})+D_{W} E_{1}\left(\hat{\Phi}, \hat{W}, \hat{I}_{e}\right)(W(s)-\hat{W})\right\rangle$

$$
+\left\langle P_{1}, D_{I_{e}} E_{1}\left(\hat{\Phi}, \hat{W}, \hat{I}_{e}\right)\left(I_{e}-\hat{I}_{e}\right)\right\rangle=0 ;
$$

$\lim _{s \rightarrow 0+0} \frac{1}{s}\left\langle P_{2}, D_{\Phi} E_{2}(\hat{\Phi}, \hat{W})(\Phi(s)-\hat{\Phi})+D_{W} E_{2}(\hat{\Phi}, \hat{W})(W(s)-\hat{W})\right\rangle=0$.

Proof. The proof relies on the principal theorem of the calculus in its Bochner integral version, ${ }^{21)}$ which becomes applicable due to our assumptions about the differentiability of $r$. We start with the feasibility of $\left(\Phi(s), W(s), I_{e}(s)\right)$ and $\left(\hat{\Phi}, \hat{W}, \hat{I}_{e}\right)$ :

$$
\begin{aligned}
\mathfrak{o}= & E_{1}\left(\Phi(s), W(s), I_{e}(s)\right)-E_{1}\left(\hat{\Phi}, \hat{W}, \hat{I}_{e}\right)=\int_{0}^{1} D_{\left(\Phi, W, I_{e}\right)} E_{1}(\hat{\Phi}+\tau(\Phi(s)-\hat{\Phi}), \\
& \left.\hat{W}+\tau(W(s)-\hat{W}), \hat{I}_{e}+\tau\left(I_{e}(s)-\hat{I}_{e}\right)\right)\left(\Phi(s)-\hat{\Phi}, W(s)-\hat{W}, I_{e}(s)-\hat{I}_{e}\right) d \tau \Longrightarrow \\
0= & \left\langle P_{1}, \int_{0}^{1}\left(D_{\left(\Phi, W, I_{e}\right)} E_{1}\left(\hat{\Phi}+\tau(\Phi(s)-\hat{\Phi}), \hat{W}+\tau(W(s)-\hat{W}), \hat{I}_{e}+\tau\left(I_{e}(s)-\hat{I}_{e}\right)\right)(\Phi(s)-\hat{\Phi},\right.\right. \\
& \left.\left.\left.W(s)-\hat{W}, I_{e}(s)-\hat{I}_{e}\right)-D_{\left(\Phi, W, I_{e}\right)} E_{1}\left(\hat{\Phi}, \hat{W}, \hat{I}_{e}\right)\left(\Phi(s)-\hat{\Phi}, W(s)-\hat{W}, I_{e}(s)-\hat{I}_{e}\right)\right) d \tau\right\rangle \\
& +\left\langle P_{1}, D_{\left(\Phi, W, I_{e}\right)} E_{1}\left(\hat{\Phi}, \hat{W}, \hat{I}_{e}\right)\left(\Phi(s)-\hat{\Phi}, W(s)-\hat{W}, I_{e}(s)-\hat{I}_{e}\right)\right\rangle \\
= & \left\langle P_{1}, \int_{0}^{1}\left(D_{\Phi} E_{1}\left(\hat{\Phi}+\tau \ldots, \hat{W}+\tau \ldots, \hat{I}_{e}+\tau \ldots\right)(\Phi(s)-\hat{\Phi})-D_{\Phi} E_{1}\left(\hat{\Phi}, \hat{W}, \hat{I}_{e}\right)(\Phi(s)-\hat{\Phi})\right.\right. \\
& +D_{W} E_{1}\left(\hat{\Phi}+\tau \ldots, \hat{W}+\tau \ldots, \hat{I}_{e}+\tau \ldots\right)(W(s)-\hat{W})-D_{W} E_{1}\left(\hat{\Phi}, \hat{W}, \hat{I}_{e}\right)(W(s)-\hat{W}) \\
& \left.\left.+D_{I_{e}} E_{1}\left(\hat{\Phi}+\tau \ldots, \hat{W}+\tau \ldots, \hat{I}_{e}+\tau \ldots\right)\left(I_{e}(s)-\hat{I}_{e}\right)-D_{I_{e}} E_{1}\left(\hat{\Phi}, \hat{W}, \hat{I}_{e}\right)\left(I_{e}(s)-\hat{I}_{e}\right)\right) d \tau\right\rangle \\
+\langle & \left.P_{1}, D_{\Phi} E_{1}\left(\hat{\Phi}, \hat{W}, \hat{I}_{e}\right)(\Phi(s)-\hat{\Phi})+D_{W} E_{1}\left(\hat{\Phi}, \hat{W}, \hat{I}_{e}\right)(W(s)-\hat{W})+D_{I_{e}} E_{1}\left(\hat{\Phi}, \hat{W}, \hat{I}_{e}\right)\left(I_{e}(s)-\hat{I}_{e}\right)\right\rangle .
\end{aligned}
$$

Observe now that ${ }^{22)}$

$$
\left|\left\langle P_{1}, \int_{0}^{1}(\ldots) d \tau\right\rangle\right| \leqslant\left\|P_{1}\right\|_{Z_{1}^{*}} \cdot\left\|\int_{0}^{1}(\ldots) d \tau\right\|_{Z_{1}} \leqslant\left\|P_{1}\right\|_{Z_{1}^{*}} \cdot \int_{0}^{1}\|\ldots\|_{Z_{1}} d \tau .
$$

Consequently, for the first term within the last equation, we have

$$
\begin{aligned}
& \lim _{s \rightarrow 0+0} \frac{1}{s}\left|\left\langle P_{1}, \int_{0}^{1}(\ldots) d \tau\right\rangle\right| \leqslant \lim _{s \rightarrow 0+0}\left\|P_{1}\right\|_{\mathrm{Z}_{1}^{*}}\left(\int_{0}^{1} \mid D_{\Phi} E_{1}\left(\hat{\Phi}+\tau \ldots, \hat{W}+\tau \ldots, \hat{I}_{e}+\tau \ldots\right)\right. \\
& -D_{\Phi} E_{1}\left(\hat{\Phi}, \hat{W}, \hat{I}_{e}\right)\left\|_{\mathcal{L}\left(\widetilde{\mathrm{X}}_{1}, \mathrm{Z}_{1}\right)} \frac{1}{s}\right\| \Phi(s)-\hat{\Phi} \|_{\widetilde{\mathrm{X}}_{1}} d \tau \\
& +\int_{0}^{1}\left\|D_{W} E_{1}\left(\hat{\Phi}+\tau \ldots, \hat{W}+\tau \ldots, \hat{I}_{e}+\tau \ldots\right)-D_{W} E_{1}\left(\hat{\Phi}, \hat{W}, \hat{I}_{e}\right)\right\|_{\mathcal{L}\left(\widetilde{\mathrm{X}}_{2}, Z_{1}\right)} \frac{1}{s}\|W(s)-\hat{W}\|_{\widetilde{\mathrm{X}}_{2}} d \tau \\
& \left.+\int_{0}^{1}\left\|D_{I_{e}} E_{1}\left(\hat{\Phi}+\tau \ldots, \hat{W}+\tau \ldots, \hat{I}_{e}+\tau \ldots\right)-D_{I_{e}} E_{1}\left(\hat{\Phi}, \hat{W}, \hat{I}_{e}\right)\right\|_{\mathcal{L}\left(\mathrm{X}_{3}, Z_{1}\right)} \frac{1}{s}\left\|I_{e}(s)-\hat{I}_{e}\right\|_{\mathrm{X}_{3}} d \tau\right) \\
& \leqslant \lim _{s \rightarrow 0+0}\left\|P_{1}\right\|_{\mathrm{Z}_{1}^{*}}\left(\int_{0}^{1} L_{1} \tau\left(\|\Phi(s)-\hat{\Phi}\|_{\widetilde{\mathrm{X}}_{1}}+\|W(s)-\hat{W}\|_{\widetilde{\mathrm{X}}_{2}}+\left\|I_{e}(s)-\hat{I}_{e}\right\|_{\mathrm{X}_{3}}\right) \frac{1}{s}\|\Phi(s)-\hat{\Phi}\|_{\widetilde{\mathrm{X}}_{1}} d \tau\right. \\
& +\int_{0}^{1} L_{2} \tau\left(\|\Phi(s)-\hat{\Phi}\|_{\widetilde{\mathrm{X}}_{1}}+\|W(s)-\hat{W}\|_{\widetilde{\mathrm{X}}_{2}}+\left\|I_{e}(s)-\hat{I}_{e}\right\|_{\mathrm{X}_{3}}\right) \frac{1}{s}\|W(s)-\hat{W}\|_{\widetilde{\mathrm{X}}_{2}} d \tau \\
& \left.+\int_{0}^{1} L_{3} \tau\left(\|\Phi(s)-\hat{\Phi}\|_{\widetilde{\mathrm{X}}_{1}}+\|W(s)-\hat{W}\|_{\widetilde{\mathrm{X}}_{2}}+\left\|I_{e}(s)-\hat{I}_{e}\right\|_{\mathrm{X}_{3}}\right) \frac{1}{s}\left\|I_{e}(s)-\hat{I}_{e}\right\|_{\mathrm{X}_{3}} d \tau\right)
\end{aligned}
$$

21) [BERGER 77], p. 68, (2.1.11).

22) For the last inequality, cf. [Yosida 95], p. 133, Corollary 1. 
with Lipschitz constants $L_{1}, L_{2}, L_{3}$, whose existence is ensured by the twice continuous Fréchet differentiability of $E_{1}$ with respect to $\Phi, W$ and $I_{e}$. With Lemma 3.11., we may further estimate:

$$
\begin{aligned}
& \lim _{s \rightarrow 0+0} \frac{1}{s}\left|\left\langle P_{1}, \int_{0}^{1}(\ldots) d \tau\right\rangle\right| \\
& \leqslant \lim _{s \rightarrow 0+0}\left\|P_{1}\right\|_{\mathrm{Z}_{1}^{*}} \frac{1}{2}\left(L_{1}+L_{2}+L_{3}\right) \frac{1}{s}\left(\|\Phi(s)-\hat{\Phi}\|_{\widetilde{\mathrm{X}}_{1}}+\|W(s)-\hat{W}\|_{\widetilde{\mathrm{X}}_{2}}+\left\|I_{e}(s)-\hat{I}_{e}\right\|_{\mathrm{X}_{3}}\right)^{2} \\
& \leqslant \lim _{s \rightarrow 0+0}\left\|P_{1}\right\|_{\mathrm{Z}_{1}^{*}} C\left(\frac{1}{s}\|\Phi(s)-\hat{\Phi}\|_{\widetilde{\mathrm{X}}_{1}}^{2}+\frac{1}{s}\|W(s)-\hat{W}\|_{\widetilde{\mathrm{X}}_{2}}^{2}+\frac{1}{s}\left\|I_{e}(s)-\hat{I}_{e}\right\|_{\mathrm{X}_{3}}^{2}\right)=0
\end{aligned}
$$

which implies that

$$
\begin{aligned}
\lim _{s \rightarrow 0+0} \frac{1}{s}\left\langle P_{1}, D_{\Phi} E_{1}\left(\hat{\Phi}, \hat{W}, \hat{I}_{e}\right)(\Phi(s)-\hat{\Phi})+D_{W} E_{1}\left(\hat{\Phi}, \hat{W}, \hat{I}_{e}\right)(W(s)-\hat{W})\right. & \\
+ & \left.+D_{I_{e}} E_{1}\left(\hat{\Phi}, \hat{W}, \hat{I}_{e}\right)\left(I_{e}(s)-\hat{I}_{e}\right)\right\rangle=0 .
\end{aligned}
$$

Analogously, the following equation holds:

$$
\begin{aligned}
\mathfrak{o}= & E_{2}(\Phi(s), W(s))-E_{2}(\hat{\Phi}, \hat{W}) \\
= & \int_{0}^{1} D_{(\Phi, W)} E_{2}(\hat{\Phi}+\tau(\Phi(s)-\hat{\Phi}), \hat{W}+\tau(W(s)-\hat{W}))(\Phi(s)-\hat{\Phi}, W(s)-\hat{W}) d \tau \Longrightarrow \\
0= & \left\langle P_{2}, \int_{0}^{1}\left(D_{(\Phi, W)} E_{2}(\hat{\Phi}+\tau(\Phi(s)-\hat{\Phi}), \hat{W}+\tau(W(s)-\hat{W}))(\Phi(s)-\hat{\Phi}, W(s)-\hat{W})\right.\right. \\
& \left.\left.-D_{(\Phi, W)} E_{2}(\hat{\Phi}, \hat{W})(\Phi(s)-\hat{\Phi}, W(s)-\hat{W})\right) d \tau\right\rangle+\left\langle P_{2}, D_{(\Phi, W)} E_{2}(\hat{\Phi}, \hat{W})(\Phi(s)-\hat{\Phi}, W(s)-\hat{W})\right\rangle \\
= & \left\langle P_{2}, \int_{0}^{1}\left(D_{\Phi} E_{2}(\hat{\Phi}+\tau \ldots, \hat{W}+\tau \ldots)(\Phi(s)-\hat{\Phi})-D_{\Phi} E_{2}(\hat{\Phi}, \hat{W})(\Phi(s)-\hat{\Phi})\right.\right. \\
& \left.\left.+D_{W} E_{2}(\hat{\Phi}+\tau \ldots, \hat{W}+\tau \ldots)(W(s)-\hat{W})-D_{W} E_{2}(\hat{\Phi}, \hat{W})(W(s)-\hat{W})\right) d \tau\right\rangle \\
& +\left\langle P_{2}, D_{\Phi} E_{2}(\hat{\Phi}, \hat{W})(\Phi(s)-\hat{\Phi})+D_{W} E_{2}(\hat{\Phi}, \hat{W})(W(s)-\hat{W})\right\rangle
\end{aligned}
$$

For the first term, we find

$$
\begin{aligned}
& \lim _{s \rightarrow 0+0} \frac{1}{s} \mid\left\langle P_{2}, \int_{0}^{1}(\ldots) d \tau\right| \\
\leqslant & \lim _{s \rightarrow 0+0}\left\|P_{2}\right\|_{\mathrm{Z}_{2}^{*}}\left(\int_{0}^{1}\left\|D_{\Phi} E_{2}(\hat{\Phi}+\tau \ldots, \hat{W}+\tau \ldots)-D_{\Phi} E_{2}(\hat{\Phi}, \hat{W})\right\|_{\mathcal{L}\left(\widetilde{\mathrm{X}}_{1}, \mathrm{Z}_{2}\right)} \frac{1}{s}\|\Phi(s)-\hat{\Phi}\|_{\widetilde{\mathrm{X}}_{1}} d \tau\right. \\
& \left.+\int_{0}^{1}\left\|D_{W} E_{2}(\hat{\Phi}+\tau \ldots, \hat{W}+\tau \ldots)-D_{W} E_{2}(\hat{\Phi}, \hat{W})\right\|_{\mathcal{L}\left(\widetilde{\mathrm{X}}_{2}, Z_{2}\right)} \frac{1}{s}\|W(s)-\hat{W}\|_{\widetilde{\mathrm{X}}_{2}} d \tau\right) \\
\leqslant & \lim _{s \rightarrow 0+0}\left\|P_{2}\right\|_{\mathrm{Z}_{2}^{*}}\left(\int_{0}^{1} L_{4} \tau\left(\|\Phi(s)-\hat{\Phi}\|_{\widetilde{\mathrm{X}}_{1}}+\|W(s)-\hat{W}\|_{\widetilde{\mathrm{X}}_{2}}\right) \frac{1}{s}\|\Phi(s)-\hat{\Phi}\|_{\widetilde{\mathrm{X}}_{1}} d \tau\right. \\
& \left.+\int_{0}^{1} L_{5} \tau\left(\|\Phi(s)-\hat{\Phi}\|_{\widetilde{\mathrm{X}}_{1}}+\|W(s)-\hat{W}\|_{\widetilde{\mathrm{X}}_{2}}\right) \frac{1}{s}\|W(s)-\hat{W}\|_{\widetilde{\mathrm{X}}_{2}} d \tau\right)
\end{aligned}
$$

with Lipschitz constants $L_{4}, L_{5}$, whose existence follows from the twice continuous Fréchet differentiability of $E_{2}$ with respect to $\Phi$ and $W$. Thus the estimate (3.104) may be continued as follows:

$$
\begin{aligned}
& \lim _{s \rightarrow 0+0} \frac{1}{s}\left|\left\langle P_{2}, \int_{0}^{1}(\ldots) d \tau\right\rangle\right| \leqslant \lim _{s \rightarrow 0+0}\left\|P_{2}\right\|_{\mathrm{Z}_{2}^{*}} \frac{1}{2}\left(L_{4}+L_{5}\right) \frac{1}{s}\left(\|\Phi(s)-\hat{\Phi}\|_{\widetilde{\mathrm{X}}_{1}}+\|W(s)-\hat{W}\|_{\widetilde{\mathrm{X}}_{2}}\right)^{2} \\
& \leqslant \lim _{s \rightarrow 0+0}\left\|P_{2}\right\|_{\mathrm{Z}_{2}^{*}} C\left(\frac{1}{s}\|\Phi(s)-\hat{\Phi}\|_{\widetilde{\mathrm{X}}_{1}}^{2}+\frac{1}{s}\|W(s)-\hat{W}\|_{\widetilde{\mathrm{X}}_{2}}^{2}\right)=0
\end{aligned}
$$


This implies that

$$
\lim _{s \rightarrow 0+0} \frac{1}{s}\left\langle P_{2}, D_{\Phi} E_{2}(\hat{\Phi}, \hat{W})(\Phi(s)-\hat{\Phi})+D_{W} E_{2}(\hat{\Phi}, \hat{W})(W(s)-\hat{W})\right\rangle=0
$$

- Step 4. Choose now $\varepsilon>0$ small enough in order to ensure that the difference $F\left(\Phi(s), W(s), I_{e}(s)\right)-$ $F\left(\hat{\Phi}, \hat{W}, \hat{I}_{e}\right)$ of the objective values is nonnegative for all triples $\left(\Phi(s), W(s), I_{e}(s)\right) \in \mathrm{K}(\hat{\Phi}, C \varepsilon) \times \mathrm{K}(\hat{W}, C \varepsilon) \times$ $\mathrm{K}\left(\hat{I}_{e}, \varepsilon\right)$. As a consequence of our analytical assumptions about the integrand $r$, the first variation may be written as

$$
\begin{aligned}
0 & \leqslant \delta^{+} F\left(\hat{\Phi}, \hat{W}, \hat{I}_{e}\right)\left(\Phi(1)-\hat{\Phi}, W(1)-\hat{W}, I_{e}-\hat{I}_{e}\right)=\lim _{s \rightarrow 0+0} \frac{1}{s}\left(F\left(\Phi(s), W(s), I_{e}(s)\right)-F\left(\hat{\Phi}, \hat{W}, \hat{I}_{e}\right)\right) \\
& =\lim _{s \rightarrow 0+0} \frac{1}{s}\left(D_{\Phi} F\left(\hat{\Phi}, \hat{W}, \hat{I}_{e}\right)(\Phi(s)-\hat{\Phi})+D_{W} F\left(\hat{\Phi}, \hat{W}, \hat{I}_{e}\right)(W(s)-\hat{W})+D_{I_{e}} F\left(\hat{\Phi}, \hat{W}, \hat{I}_{e}\right)\left(I_{e}(s)-\hat{I}_{e}\right)\right) .
\end{aligned}
$$

Together with Lemma 3.12., we obtain

$$
\begin{aligned}
0 \leqslant & \lim _{s \rightarrow 0+0} \frac{1}{s}\left(D_{\Phi} F\left(\hat{\Phi}, \hat{W}, \hat{I}_{e}\right)(\Phi(s)-\hat{\Phi})\right. \\
& \quad+\left\langle P_{1}, D_{\Phi} E_{1}\left(\hat{\Phi}, \hat{W}, \hat{I}_{e}\right)(\Phi(s)-\hat{\Phi})\right\rangle+\left\langle P_{2}, D_{\Phi} E_{2}(\hat{\Phi}, \hat{W})(\Phi(s)-\hat{\Phi})\right\rangle \\
& +D_{W} F\left(\hat{\Phi}, \hat{W}, \hat{I}_{e}\right)(W(s)-\hat{W}) \\
& +\left\langle P_{1}, D_{W} E_{1}\left(\hat{\Phi}, \hat{W}, \hat{I}_{e}\right)(W(s)-\hat{W})\right\rangle+\left\langle P_{2}, D_{W} E_{2}(\hat{\Phi}, \hat{W})(W(s)-\hat{W})\right\rangle \\
& \left.+D_{I_{e}} F\left(\hat{\Phi}, \hat{W}, \hat{I}_{e}\right)\left(I_{e}(s)-\hat{I}_{e}\right)+\left\langle P_{1}, D_{I_{e}} E_{1}\left(\hat{\Phi}, \hat{W}, \hat{I}_{e}\right)\left(I_{e}-\hat{I}_{e}\right)\right\rangle\right)
\end{aligned}
$$

where the first two parts vanish since $P_{1}, P_{2}$ solve the adjoint equations

$$
\begin{aligned}
& D_{\Phi} F\left(\hat{\Phi}, \hat{W}, \hat{I}_{e}\right)+\left\langle P_{1}, D_{\Phi} E_{1}\left(\hat{\Phi}, \hat{W}, \hat{I}_{e}\right)\right\rangle+\left\langle P_{2}, D_{\Phi} E_{2}(\hat{\Phi}, \hat{W})\right\rangle=0 \text { and } \\
& D_{W} F\left(\hat{\Phi}, \hat{W}, \hat{I}_{e}\right)+\left\langle P_{1}, D_{W} E_{1}\left(\hat{\Phi}, \hat{W}, \hat{I}_{e}\right)\right\rangle+\left\langle P_{2}, D_{W} E_{2}(\hat{\Phi}, \hat{W})\right\rangle=0 .
\end{aligned}
$$

Note that, by Subsection 3.c) above, these equations take the claimed form. Consequently, we arrive at

$$
\begin{aligned}
0 & \leqslant \lim _{s \rightarrow 0+0} \frac{1}{s}\left(D_{I_{e}} F\left(\hat{\Phi}, \hat{W}, \hat{I}_{e}\right)\left(I_{e}(s)-\hat{I}_{e}\right)+\left\langle P_{1}, D_{I_{e}} E_{1}\left(\hat{\Phi}, \hat{W}, \hat{I}_{e}\right)\left(I_{e}-\hat{I}_{e}\right)\right\rangle\right) \\
& =\int_{0}^{T} \int_{\Omega}\left(\mu \hat{I}_{e}(x, t)+\frac{1}{1+\lambda} P_{1}(x, t)\right)\left(I_{e}(x, t)-\hat{I}_{e}(x, t)\right) d x d t .
\end{aligned}
$$

This implies the claimed optimality condition (3.23), and the proof is complete.

Proof of Corollary 3.6. The non-Lebesgue points of $\hat{I}_{e}, P_{1},{ }^{23)}$

$$
\left(\mu(1+\lambda) \hat{I}_{e}(x, t)+P_{1}(x, t)\right) \hat{I}_{e}(x, t) \quad \text { and } \quad\left(\mu(1+\lambda) \hat{I}_{e}(x, t)+P_{1}(x, t)\right) \eta
$$

form null sets for arbitrary $\eta \in[-R, R]$ since the set of Lebesgue points is conserved under linear combinations. Assumption a) of Theorem 3.5. implies that the boundary $\partial \Omega \times[0, T]$ of $\Omega_{T}$ forms a null set as well. ${ }^{24)}$ Denote by $\mathrm{N}$ the union of all these subsets, which is still a Lebesgue null set, and consider a point

23) For the following arguments, cf. [WAGner 09] , p. 553 f., Proof of Theorem 2.3.

24) [WAGNer 06] , p. 122, Lemma 9.2. 
$\left(x_{0}, t_{0}\right) \in \operatorname{int}\left(\Omega_{T}\right) \backslash \mathrm{N}$ and a number $\eta_{0} \in[-R, R]$. From a Vitali covering of $\Omega_{T},{ }^{25)}$ choose some decreasing sequence $\left\{\mathrm{E}^{N}\right\}$ of closed subsets of $\Omega_{T}$ with $\bigcap_{N} \mathrm{E}^{N}=\left\{\left(x_{0}, t_{0}\right)\right\}$. Together with $\hat{I}_{e}$, all functions

$$
I^{N}(x, t)=\mathbb{1}_{\mathrm{E}^{N}}(x, t) \cdot \eta_{0}+\mathbb{1}_{\left(\Omega \backslash \mathrm{E}^{N}\right)}(x, t) \cdot \hat{I}_{e}(x, t)
$$

belong to $\mathcal{C} \subset L^{\infty}\left(\Omega_{T}\right) \subset L^{\infty}\left[(0, T), L^{2}(\Omega)\right]$. Consequently, we may form the Lebesgue derivative

$$
\begin{aligned}
\lim _{N \rightarrow \infty} & \frac{1}{\left|\mathrm{E}^{N}\right|} \int_{0}^{T} \int_{\Omega}\left(\mu(1+\lambda) \hat{I}_{e}(x, t)+P_{1}(x, t)\right)\left(I^{N}(x, t)-\hat{I}_{e}(x, t)\right) d x d t \\
\quad= & \lim _{N \rightarrow \infty} \frac{1}{\left|\mathrm{E}^{N}\right|} \int_{\mathrm{E}^{N}}\left(\mu(1+\lambda) \hat{I}_{e}(x, t)+P_{1}(x, t)\right)\left(\eta_{0}-\hat{I}_{e}(x, t)\right) d(x, t) \\
\quad= & \left(\mu(1+\lambda) \hat{I}_{e}\left(x_{0}, t_{0}\right)+P_{1}\left(x_{0}, t_{0}\right)\right)\left(\eta_{0}-\hat{I}_{e}\left(x_{0}, t_{0}\right)\right) \geqslant 0,
\end{aligned}
$$

and this implies the claimed conditions (3.24) and (3.25).

Proof of Corollary 3.7. If $\left(\hat{\Phi}, \hat{W}, \hat{I}_{e}\right)$ is a weak local minimizer of $(\mathrm{P})$ with $\operatorname{ess} \sup _{(x, t) \in \Omega_{T}}\left|\hat{I}_{e}(x, t)\right|<R$ then (3.25) implies that

$$
\hat{I}_{e}(x, t)=\frac{1}{\mu(1+\lambda)} P_{1}(x, t)
$$

for almost all $(x, t) \in \Omega_{T}$, and $\hat{I}_{e}$ and $P_{1}$ belong to the same space $W^{1,2}\left[(0, T), L^{2}(\Omega)\right] \cap L^{2}[(0, T)$, $\left.W^{2,2}(\Omega)\right]$.

\section{Appendix I: Bochner integrable mappings.}

\section{a) Survey of spaces of Bochner integrable functions.}

Let $\mathrm{X}$ be a Banach space. Then a mapping $f:[0, T] \rightarrow \mathrm{X}$ is called strongly measurable iff there is a sequence $\left\{f^{N}\right\}$ of simple mappings $f^{N}:[0, T] \rightarrow \mathrm{X}$ of the form $f^{N}(t)=\sum_{k=1}^{K} \mathbb{1}_{\mathrm{A}_{k}}(t) x_{k}$ with $x_{k} \in \mathrm{X}$ and Lebesgue subsets $\mathrm{A}_{k} \subseteq[0, T]$ such that $\left\|f^{N}(t)-f(t)\right\|_{\mathrm{X}} \rightarrow 0$ for almost all $t \in[0, T] .{ }^{26)}$

Definition 4.1. (Bochner function spaces) 1) Let $1 \leqslant p<\infty$. Then the space $L^{p}[(0, T), \mathrm{X}]$ consists of all strongly measurable functions $f:[0, T] \rightarrow \mathrm{X}$ with

$$
\|f\|_{L^{p}[(0, T), \mathrm{X}]}=\left(\int_{0}^{T}\|f(t)\|_{\mathrm{X}} d t\right)^{1 / p}<\infty .
$$

2) The space $L^{\infty}[(0, T), \mathrm{X}]$ consists of all strongly measurable functions $f:[0, T] \rightarrow \mathrm{X}$ with

$$
\|f\|_{L^{\infty}[(0, T), \mathrm{X}]}=\operatorname{ess}_{\sup } \operatorname{si0,T]}_{t \in f(t) \|_{\mathrm{X}}<\infty .}
$$

3) The space $W^{1, p}[(0, T), \mathrm{X}]$ consists of all functions $f \in L^{p}[(0, T), \mathrm{X}]$, admitting a weak derivative $d f / d t$, which belongs to $L^{p}[(0, T), \mathrm{X}]$ as well. The weak derivative is defined by the usual formula wherein the integrals are interpreted in the Bochner sense.

25) [Dunford/Schwartz 88], p. 212, Definition 2.

26) Here and in Definition 4.1., we follow [Evans 98], pp. 285 f. and 649 f. 
4) The space $C^{0}[[0, T], \mathrm{X}]$ contains all continuous mappings $f:[0, T] \rightarrow \mathrm{X}$ with

$$
\|f\|_{C^{0}[[0, T], \mathrm{X}]}=\operatorname{Max}_{t \in[0, T]}\|f(t)\|_{\mathrm{X}}<\infty \text {. }
$$

\section{b) Imbedding theorems for Bochner spaces.}

Proposition 4.2. Assume that $\Omega \subset \mathbb{R}^{m}$ is compact and $1 \leqslant p, q \leqslant \infty$. Then $L^{p}\left[(0, T), L^{q}(\Omega)\right]$ is continuously imbedded into $L^{\operatorname{Min}(p, q)}\left(\Omega_{T}\right)$.

Proof. This is a consequence of [Elstrodt 96] , p. 232, Theorem 2.10.

Proposition 4.3. ${ }^{27)}$ If $1 \leqslant p \leqslant q<\infty$ then $L^{p}\left[\Omega, L^{q}(0, T)\right]$ is continuously imbedded into $L^{q}[(0, T)$, $\left.L^{p}(\Omega)\right]$.

Proposition 4.4. If $1 \leqslant p<q \leqslant \infty$ and $\mathrm{X}$ is a Banach space then $L^{q}[(0, T)$, $\mathrm{X}]$ is continuously imbedded into $L^{p}[(0, T), \mathrm{X}]$.

Proof. Follows from [Elstrodt 96], p. 232, Theorem 2.10., as well.

Theorem 4.5. ${ }^{28)}$ If $1 \leqslant p \leqslant \infty$ and $\mathrm{X}$ is a Banach space then $W^{1, p}[(0, T), \mathrm{X}]$ is continuously imbedded into $C^{0}[[0, T], \mathrm{X}]$.

Theorem 4.6. (Aubin-Dubinskij lemma) ${ }^{29)}$ Consider three normed spaces $\mathrm{X}_{0} \subseteq \mathrm{X} \subseteq \mathrm{X}_{1}$ where the imbedding $\mathrm{X}_{0} \hookrightarrow \mathrm{X}$ is compact and the imbedding $\mathrm{X} \hookrightarrow \mathrm{X}_{1}$ is continuous. If $p, p^{\prime} \in(1, \infty)$ then the space

$$
\mathrm{Y}=\left\{f \in L^{p}\left[(0, T), \mathrm{X}_{0}\right] \mid \frac{d f}{d t} \in L^{p^{\prime}}\left[(0, T), \mathrm{X}_{1}\right]\right\}
$$

is compactly imbedded into $L^{q}[(0, T), \mathrm{X}]$ for arbitrary $q \in(1, \infty)$.

\section{Appendix II: Proof of the stability estimate.}

Proof of Theorem 3.8. Throughout the following proof, $C$ denotes a generical positive constant, which may appropriately change from line to line. $C$ will never depend on the data $\Phi_{0}, W_{0}, I_{i}$ and $I_{e}$ but, possibly, on $\Omega$ and $p=4$.

- Step 1. The difference of the parabolic equations. From the parabolic equations, satisfied by the pairs $\left(\Phi^{\prime}, W^{\prime}\right)$ and $\left(\Phi^{\prime \prime}, W^{\prime \prime}\right)$ for almost all $t \in[0, T]$, we obtain the difference

$$
\begin{aligned}
\left\langle\frac{d}{d t}\left(\Phi^{\prime}(t)-\Phi^{\prime \prime}(t)\right), \psi\right\rangle+M\left(\Phi^{\prime}(t)-\Phi^{\prime \prime}(t), \psi\right) & \\
\quad+\int_{\Omega}\left(I _ { i o n } \left(\Phi^{\prime}(t),\right.\right. & \left.\left.W^{\prime}(t)\right)-I_{i o n}\left(\Phi^{\prime \prime}(t), W^{\prime \prime}(t)\right)\right) \psi d x \\
& =\left\langle\frac{1}{1+\lambda}\left(\lambda\left(I_{i}{ }^{\prime}(t)-I_{i}^{\prime \prime}(t)\right)-\left(I_{e}{ }^{\prime}(t)-I_{e}^{\prime \prime}(t)\right)\right), \psi\right\rangle \quad \forall \psi \in W^{1,2}(\Omega) .
\end{aligned}
$$

Inserting into (5.1) the feasible test function $\psi=\Phi^{\prime}(t)-\Phi^{\prime \prime}(t) \in W^{1,2}(\Omega)$ and applying inequality (2.16) from Lemma 2.3., we arrive at

$$
\begin{aligned}
\frac{1}{2} \frac{d}{d t}\left\|\Phi^{\prime}-\Phi^{\prime \prime}\right\|_{L^{2}(\Omega)}^{2}+\beta & \left\|\Phi^{\prime}-\Phi^{\prime \prime}\right\|_{W^{1,2}(\Omega)}^{2}+\int_{\Omega}\left(I_{i o n}\left(\Phi^{\prime}, W^{\prime}\right)-I_{i o n}\left(\Phi^{\prime \prime}, W^{\prime \prime}\right)\right)\left(\Phi^{\prime}-\Phi^{\prime \prime}\right) d x \\
& \leqslant\left|\left\langle\frac{1}{1+\lambda}\left(\lambda\left(I_{i}{ }^{\prime}-I_{i}^{\prime \prime}\right)-\left(I_{e}{ }^{\prime}-I_{e}{ }^{\prime \prime}\right)\right), \Phi^{\prime}-\Phi^{\prime \prime}\right\rangle\right|+\beta\left\|\Phi^{\prime}-\Phi^{\prime \prime}\right\|_{L^{2}(\Omega)}^{2}
\end{aligned}
$$

27) [Garling 07], p. 52, Corollary 5.4.2.

28) [Evans 98], p. 286, Theorem 2.

29) [DubinskiJ 65], p. 612, Teorema 1, and p. 615, Teorema 2. 
The first term on the right-hand side can be estimated through

$$
\begin{aligned}
& \left|\left\langle\frac{1}{1+\lambda}\left(\lambda\left(I_{i}{ }^{\prime}(t)-I_{i}{ }^{\prime \prime}(t)\right)-\left(I_{e}{ }^{\prime}(t)-I_{e}{ }^{\prime \prime}(t)\right)\right), \Phi^{\prime}-\Phi^{\prime \prime}\right\rangle\right| \leqslant \frac{1}{2 \varepsilon_{1}} \cdot C\left(\left\|I_{i}{ }^{\prime}(t)-I_{i}{ }^{\prime \prime}(t)\right\|^{2}\left(W^{1,2}(\Omega)\right)^{*}\right. \\
& \left.+\left\|I_{e}{ }^{\prime}(t)-I_{e}^{\prime \prime}(t)\right\|_{\left(W^{1,2}(\Omega)\right)^{*}}^{2}\right)+\frac{3 \varepsilon_{1}}{4}\left\|\Phi^{\prime}(t)-\Phi^{\prime \prime}(t)\right\|_{W^{1,2}(\Omega)}^{2}
\end{aligned}
$$

with arbitrary $\varepsilon_{1}>0$. The second term will be estimated with the help of the following lemma.

Lemma 2.10. For all $\varphi_{1}, \varphi_{2} \in \mathbb{R}$, the following identity holds:

$$
\begin{aligned}
\left(\varphi_{1}^{3}-(a+1) \varphi_{1}^{2}+a \varphi_{1}\right)-\left(\varphi_{2}^{3}-\right. & \left.(a+1) \varphi_{2}^{2}+a \varphi_{2}\right) \\
& =\left(\varphi_{1}-\varphi_{2}\right) \cdot\left(\varphi_{1}^{2}+\varphi_{1} \varphi_{2}+\varphi_{2}^{2}-(a+1)\left(\varphi_{1}+\varphi_{2}\right)+a\right) .
\end{aligned}
$$

Consequently, we get

$$
\begin{gathered}
\int_{\Omega}\left(I_{\text {ion }}\left(\Phi^{\prime}, W^{\prime}\right)-I_{\text {ion }}\left(\Phi^{\prime \prime}, W^{\prime \prime}\right)\right)\left(\Phi^{\prime}-\Phi^{\prime \prime}\right) d x \\
=\int_{\Omega}\left(\Phi^{\prime}-\Phi^{\prime \prime}\right) b\left(\left(\Phi^{\prime}\right)^{2}+\Phi^{\prime} \Phi^{\prime \prime}+\left(\Phi^{\prime \prime}\right)^{2}+a\right)\left(\Phi^{\prime}-\Phi^{\prime \prime}\right) d x \\
\quad-(a+1) b \int_{\Omega}\left(\Phi^{\prime}-\Phi^{\prime \prime}\right)\left(\Phi^{\prime}+\Phi^{\prime \prime}\right)\left(\Phi^{\prime}-\Phi^{\prime \prime}\right) d x+\int_{\Omega}\left(\Phi^{\prime} W^{\prime}-\Phi^{\prime \prime} W^{\prime \prime}\right)\left(\Phi^{\prime}-\Phi^{\prime \prime}\right) d x
\end{gathered}
$$

Since $\Phi^{\prime}(x, t)^{2}+\Phi^{\prime}(x, t) \Phi^{\prime \prime}(x, t)+\Phi^{\prime \prime}(x, t)^{2} \geqslant 0$ for almost all $(x, t) \in \Omega_{T}$ and $a, b>0$, the inequalities (5.2), (5.3) and (5.5) imply

$$
\begin{array}{r}
\frac{d}{d t}\left\|\Phi^{\prime}-\Phi^{\prime \prime}\right\|_{L^{2}(\Omega)}^{2}+2 \beta\left\|\Phi^{\prime}-\Phi^{\prime \prime}\right\|_{W^{1,2}(\Omega)}^{2} \leqslant 2 C \int_{\Omega}\left|\Phi^{\prime}-\Phi^{\prime \prime}\right| \cdot\left|\Phi^{\prime}+\Phi^{\prime \prime}\right| \cdot\left|\Phi^{\prime}-\Phi^{\prime \prime}\right| d x \\
+2 \int_{\Omega}\left(\Phi^{\prime} W^{\prime}-\Phi^{\prime \prime} W^{\prime \prime}\right)\left(\Phi^{\prime \prime}-\Phi^{\prime}\right) d x+\frac{C}{\varepsilon_{1}}\left(\left\|I_{i}{ }^{\prime}-I_{i}^{\prime \prime}\right\|_{\left(W^{1,2}(\Omega)\right.}^{2}+\left\|I_{e}{ }^{*}-I_{e}^{\prime \prime}\right\|_{\left(W^{1,2}(\Omega)\right)^{*}}^{2}\right) \\
+\frac{3 \varepsilon_{1}}{2}\left\|\Phi^{\prime}-\Phi^{\prime \prime}\right\|_{W^{1,2}(\Omega)}^{2}+2 \beta\left\|\Phi^{\prime}-\Phi^{\prime \prime}\right\|_{L^{2}(\Omega)}^{2}
\end{array}
$$

Applying first the generalized Cauchy's inequality with $\varepsilon_{2}>0$ and subsequently Hölder's inequality to the first term on the right-hand side of (5.6), we get

$$
\begin{aligned}
& 2 C \int_{\Omega}\left|\Phi^{\prime}-\Phi^{\prime \prime}\right| \cdot\left|\Phi^{\prime}+\Phi^{\prime \prime}\right| \cdot\left|\Phi^{\prime}-\Phi^{\prime \prime}\right| d x \\
& \leqslant C \frac{\varepsilon_{2}}{2}\left(\int_{\Omega}\left|\Phi^{\prime}+\Phi^{\prime \prime}\right|^{4} d x\right)^{1 / 2}\left(\left\|\Phi^{\prime}-\Phi^{\prime \prime}\right\|_{L^{4}(\Omega)}^{4}\right)^{1 / 2}+\frac{C}{2 \varepsilon_{2}}\left\|\Phi^{\prime}-\Phi^{\prime \prime}\right\|_{L^{2}(\Omega)}^{2} \\
& \leqslant C \frac{\varepsilon_{2}}{2}\left(\int_{\Omega}\left|\Phi^{\prime}+\Phi^{\prime \prime}\right|^{4} d x\right)^{1 / 2}\left\|\Phi^{\prime}-\Phi^{\prime \prime}\right\|_{W^{1,2}(\Omega)}^{2}+\frac{C}{2 \varepsilon_{2}}\left\|\Phi^{\prime}-\Phi^{\prime \prime}\right\|_{L^{2}(\Omega)}^{2} .
\end{aligned}
$$

The second term on the right-hand side of (5.6) will be estimated through

$$
\begin{aligned}
& 2 \int_{\Omega}\left(\Phi^{\prime} W^{\prime}-\Phi^{\prime \prime} W^{\prime \prime}\right)\left(\Phi^{\prime \prime}-\Phi^{\prime}\right) d x \\
& \quad=2 \int_{\Omega}\left(\Phi^{\prime}-\Phi^{\prime \prime}\right) W^{\prime}\left(\Phi^{\prime}-\Phi^{\prime \prime}\right) d x+2 \int_{\Omega}\left(W^{\prime}-W^{\prime \prime}\right) \Phi^{\prime \prime}\left(\Phi^{\prime}-\Phi^{\prime \prime}\right) d x \\
& \quad \leqslant \varepsilon_{3} \int_{\Omega}\left(W^{\prime}\right)^{2}\left|\Phi^{\prime}-\Phi^{\prime \prime}\right|^{2} d x+\frac{1}{\varepsilon_{3}}\left\|\Phi^{\prime}-\Phi^{\prime \prime}\right\|_{L^{2}(\Omega)}^{2} \\
& \quad+\varepsilon_{4} \int_{\Omega}\left(\Phi^{\prime \prime}\right)^{2}\left|\Phi^{\prime}-\Phi^{\prime \prime}\right|^{2} d x+\frac{1}{\varepsilon_{4}}\left\|W^{\prime}-W^{\prime \prime}\right\|_{L^{2}(\Omega)}^{2}
\end{aligned}
$$




$$
\begin{gathered}
\leqslant \varepsilon_{3}\left(\int_{\Omega}\left(W^{\prime}\right)^{4} d x\right)^{1 / 2}\left(\left\|\Phi^{\prime}-\Phi^{\prime \prime}\right\|_{L_{(\Omega)}^{4}}^{4}\right)^{1 / 2}+\frac{1}{\varepsilon_{3}}\left\|\Phi^{\prime}-\Phi^{\prime \prime}\right\|_{L^{2}(\Omega)}^{2} \\
+\varepsilon_{4}\left(\int_{\Omega}\left(\Phi^{\prime \prime}\right)^{4} d x\right)^{1 / 2}\left(\left\|\Phi^{\prime}-\Phi^{\prime \prime}\right\|_{L^{4}(\Omega)}^{4}\right)^{1 / 2}+\frac{1}{\varepsilon_{4}}\left\|W^{\prime}-W^{\prime \prime}\right\|_{L^{2}(\Omega)}^{2} \\
\leqslant C \varepsilon_{3}\left\|W^{\prime}\right\|_{L^{4}(\Omega)}^{2}\left\|\Phi^{\prime}-\Phi^{\prime \prime}\right\|_{W^{1,2}(\Omega)}^{2}+\frac{1}{\varepsilon_{3}}\left\|\Phi^{\prime}-\Phi^{\prime \prime}\right\|_{L^{2}(\Omega)}^{2} \\
+C \varepsilon_{4}\left\|\Phi^{\prime \prime}\right\|_{L^{4}(\Omega)}^{2}\left\|\Phi^{\prime}-\Phi^{\prime \prime}\right\|_{W^{1,2}(\Omega)}^{2}+\frac{1}{\varepsilon_{4}}\left\|W^{\prime}-W^{\prime \prime}\right\|_{L^{2}(\Omega)}^{2},
\end{gathered}
$$

applying the generalized Cauchy's inequality again with $\varepsilon_{3}, \varepsilon_{4}>0$. Assembling now (5.6), (5.8) and (5.12), we arrive at

$$
\begin{aligned}
& \frac{d}{d t}\left\|\Phi^{\prime}-\Phi^{\prime \prime}\right\|_{L^{2}(\Omega)}^{2}+2 \beta\left\|\Phi^{\prime}-\Phi^{\prime \prime}\right\|_{W^{1,2}(\Omega)}^{2} \\
& \leqslant C \\
& \quad \frac{\varepsilon_{2}}{2}\left(\left\|\Phi^{\prime}\right\|_{L^{4}(\Omega)}^{4}+\left\|\Phi^{\prime \prime}\right\|_{L^{4}(\Omega)}^{4}\right)^{1 / 2}\left\|\Phi^{\prime}-\Phi^{\prime \prime}\right\|_{W^{1,2}(\Omega)}^{2}+\frac{C}{2 \varepsilon_{2}}\left\|\Phi^{\prime}-\Phi^{\prime \prime}\right\|_{L^{2}(\Omega)}^{2} \\
& +C \varepsilon_{3}\left\|W^{\prime}\right\|_{L^{4}(\Omega)}^{2}\left\|\Phi^{\prime}-\Phi^{\prime \prime}\right\|_{W^{1,2}(\Omega)}^{2}+\frac{1}{\varepsilon_{3}}\left\|\Phi^{\prime}-\Phi^{\prime \prime}\right\|_{L^{2}(\Omega)}^{2} \\
& \quad+C \varepsilon_{4}\left\|\Phi^{\prime \prime}\right\|_{L^{4}(\Omega)}^{2}\left\|\Phi^{\prime}-\Phi^{\prime \prime}\right\|_{W^{1,2}(\Omega)}^{2}+\frac{1}{\varepsilon_{4}}\left\|W^{\prime}-W^{\prime \prime}\right\|_{L^{2}(\Omega)}^{2} \\
& \quad+\frac{C}{\varepsilon_{1}}\left(\left\|I_{i}{ }^{\prime}(t)-I_{i}^{\prime \prime}(t)\right\|_{\left(W^{1,2}(\Omega)\right)^{*}}^{2}+\left\|I_{e}{ }^{\prime}(t)-I_{e}^{\prime \prime}(t)\right\|_{\left(W^{1,2}(\Omega)\right)^{*}}^{2}\right) \\
& \quad+\frac{3 \varepsilon_{1}}{2}\left\|\Phi^{\prime}-\Phi^{\prime \prime}\right\|_{W^{1,2}(\Omega)}^{2}+2 \beta\left\|\Phi^{\prime}-\Phi^{\prime \prime}\right\|_{L^{2}(\Omega)}^{2} .
\end{aligned}
$$

Analogously to [NAGAIAH/Kunisch/PLANK 09], p. 10, (33), we may derive

$$
\begin{aligned}
& \frac{\varrho \beta}{4}\|\Phi(t)\|_{W^{1,2}(\Omega)}^{2}+\int_{\Omega} a|\Phi(t)|^{p} d x \\
& \leqslant C\left(\varrho\|\Phi(0)\|_{L^{2}(\Omega)}^{2}+\|W(0)\|_{L^{2}(\Omega)}^{2}+\int_{0}^{t} c|\Omega| d \tau+C \int_{0}^{t}\left\|I_{i}(\tau)\right\|_{\left(W^{1,2}(\Omega)\right)^{*}}^{2} d \tau\right. \\
& \left.\quad+C \int_{0}^{t}\left\|I_{e}(\tau)\right\|_{\left(W^{1,2}(\Omega)\right)^{*}}^{2} d \tau\right)+c|\Omega|+\frac{\varrho C}{2 \beta}\left(\left\|I_{i}(t)\right\|_{\left(W^{1,2}(\Omega)\right)^{*}}^{2}+\left\|I_{e}(t)\right\|_{\left(W^{1,2}(\Omega)\right)^{*}}^{2}\right) .
\end{aligned}
$$

Due to (5.14), the $L^{4}$-norms of $\Phi^{\prime}(t), \Phi^{\prime \prime}(t)$ are bounded through

$$
\begin{aligned}
& \int_{\Omega} \Phi^{\prime}(t)^{4} d x \leqslant C\left(1+\varrho\left\|\Phi^{\prime}(0)\right\|_{L^{2}(\Omega)}^{2}+\left\|W^{\prime}(0)\right\|_{L^{2}(\Omega)}^{2}+C \int_{0}^{T}\left(\left\|I_{i}^{\prime}(\tau)\right\|_{\left(W^{1,2}(\Omega)\right.}^{2}\right)^{*}\right. \\
& \left.\left.+\left\|I_{e}{ }^{\prime}(\tau)\right\|_{\left(W^{1,2}(\Omega)\right)^{*}}^{2}\right) d \tau\right)+c|\Omega|+\frac{\varrho C}{2 \beta}\left(\left\|I_{i}{ }^{\prime}(t)\right\|_{\left(W^{1,2}(\Omega)\right)^{*}}^{2}+\left\|I_{e}{ }^{\prime}(t)\right\|_{\left(W^{1,2}(\Omega)\right)^{*}}^{2}\right) \\
& \leqslant C\left(1+\varrho\left\|\Phi_{0}\right\|_{L^{2}(\Omega)}^{2}+\left\|W_{0}\right\|_{L^{2}(\Omega)}^{2}+\left\|I_{i}{ }^{\prime}\right\|_{L^{2}\left[(0, T),\left(W^{1,2}(\Omega)\right)^{*}\right]}^{2}+\left\|I_{e}{ }^{\prime}\right\|_{L^{2}\left[(0, T),\left(W^{1,2}(\Omega)\right)^{*}\right]}^{2}\right. \\
& \left.+\left\|I_{i}{ }^{\prime}\right\|_{L^{\infty}\left[(0, T),\left(W^{1,2}(\Omega)\right)^{*}\right]}^{2}+\left\|I_{e}{ }^{\prime}\right\|_{L^{\infty}\left[(0, T),\left(W^{1,2}(\Omega)\right)^{*}\right]}^{2}\right) ; \\
& \int_{\Omega} \Phi^{\prime \prime}(t)^{4} d x \leqslant C\left(1+\varrho\left\|\Phi^{\prime \prime}(0)\right\|_{L^{2}(\Omega)}^{2}+\left\|W^{\prime \prime}(0)\right\|_{L^{2}(\Omega)}^{2}+C \int_{0}^{T}\left(\left\|I_{i}^{\prime \prime}(\tau)\right\|_{\left(W^{1,2}(\Omega)\right.}^{2}\right)^{*}\right. \\
& \left.\left.+\left\|I_{e}^{\prime \prime}(\tau)\right\|_{\left(W^{1,2}(\Omega)\right)^{*}}^{2}\right) d \tau\right)+c|\Omega|+\frac{\varrho C}{2 \beta}\left(\left\|I_{i}^{\prime \prime}(t)\right\|_{\left(W^{1,2}(\Omega)\right)^{*}}^{2}+\left\|I_{e}^{\prime \prime}(t)\right\|_{\left(W^{1,2}(\Omega)\right)^{*}}^{2}\right) \\
& \leqslant C\left(1+\varrho\left\|\Phi_{0}\right\|_{L^{2}(\Omega)}^{2}+\left\|W_{0}\right\|_{L^{2}(\Omega)}^{2}+\left\|I_{i}^{\prime \prime}\right\|_{L^{2}\left[(0, T),\left(W^{1,2}(\Omega)\right)^{*}\right]}^{2}+\left\|I_{e}^{\prime \prime}\right\|_{L^{2}\left[(0, T),\left(W^{1,2}(\Omega)\right)^{*}\right]}^{2}\right. \\
& \left.+\left\|I_{i}{ }^{\prime \prime}\right\|_{L^{\infty}\left[(0, T),\left(W^{1,2}(\Omega)\right)^{*}\right]}^{2}+\left\|I_{e}{ }^{\prime \prime}\right\|_{L^{\infty}\left[(0, T),\left(W^{1,2}(\Omega)\right)^{*}\right]}^{2}\right) \text {. }
\end{aligned}
$$


Here the assumed $L^{\infty}$-regularity of the excitations $I_{i}{ }^{\prime}, I_{e}{ }^{\prime}, I_{i}{ }^{\prime \prime}$ and $I_{e}{ }^{\prime \prime}$ has been used. As the unique (weak or strong) solution of the initial value problem $(1.7),(1.9), W^{\prime}$ admits the representation ${ }^{30)}$

$$
W^{\prime}(x, t)=W_{0}(x) e^{-\varepsilon t}+\varepsilon \kappa e^{-\varepsilon t} \int_{0}^{t} \Phi^{\prime}(x, \tau) e^{\varepsilon \tau} d \tau,
$$

consequently, it belongs to the space $C^{1}\left[(0, T), L^{2}(\Omega)\right] \cap C^{0}\left[[0, T], L^{2}(\Omega)\right]$. Together with Theorem 2.6., the $L^{4}$-norm of $W^{\prime}(t)$ can be estimated through

$$
\begin{gathered}
\int_{\Omega} W^{\prime}(t)^{4} d x \leqslant C\left\|W_{0}\right\|_{L^{4}(\Omega)}^{4}+C \varepsilon \kappa\left\|\Phi^{\prime}\right\|_{L^{4}\left(\Omega_{T}\right)}^{4} \\
\leqslant C\left\|W_{0}\right\|_{L^{4}(\Omega)}^{4}+C \varepsilon \kappa\left(1+\left\|\Phi_{0}\right\|_{L^{2}(\Omega)}^{2}+\left\|W_{0}\right\|_{L^{2}(\Omega)}^{2}\right. \\
\left.\quad+\left\|I_{i}^{\prime}\right\|_{L^{2}\left[(0, T),\left(W^{1,2}(\Omega)\right)^{*}\right]}^{2}+\left\|I_{e}{ }^{\prime}\right\|_{L^{2}\left[(0, T),\left(W^{1,2}(\Omega)\right)^{*}\right]}^{2}\right) .
\end{gathered}
$$

Inserting now (5.16), (5.18) and (5.21) into (5.13), we may fix the numbers $\varepsilon_{1}, \varepsilon_{2}, \varepsilon_{3}, \varepsilon_{4}>0$ in such a way that the terms with $\left\|\Phi^{\prime}-\Phi^{\prime \prime}\right\|_{W^{1,2}(\Omega)}^{2}$ will be annihilated. We arrive at

$$
\begin{aligned}
\frac{d}{d t} \| \Phi^{\prime}-\Phi^{\prime \prime} & \left\|_{L^{2}(\Omega)}^{2} \leqslant\left(\frac{C}{2 \varepsilon_{2}}+\frac{1}{\varepsilon_{3}}+2 \beta\right)\right\| \Phi^{\prime}-\Phi^{\prime \prime} \|_{L^{2}(\Omega)}^{2} \\
& +\frac{1}{\varepsilon_{4}}\left\|W^{\prime}-W^{\prime \prime}\right\|_{L^{2}(\Omega)}^{2}+\frac{C}{\varepsilon_{1}}\left(\left\|I_{i}^{\prime}(t)-I_{i}^{\prime \prime}(t)\right\|_{\left.\left.\left(W^{1,2}(\Omega)\right)^{*}+\left\|I_{e}{ }^{\prime}(t)-I_{e}^{\prime \prime}(t)\right\|_{\left(W^{1,2}(\Omega)\right.}^{2}\right)^{*}\right) .}\right.
\end{aligned}
$$

- Step 2. The difference of the gating equations. Inserting into the difference of the gating equations for $\left(\Phi^{\prime}, W^{\prime}\right)$ and $\left(\Phi^{\prime \prime}, W^{\prime \prime}\right)$

$$
\left\langle\frac{d}{d t}\left(W^{\prime}(t)-W^{\prime \prime}(t)\right), \psi\right\rangle=-\varepsilon \int_{\Omega}\left(W^{\prime}(t)-W^{\prime \prime}(t)\right) \psi d x+\varepsilon \kappa \int_{\Omega}\left(\Phi^{\prime}(t)-\Phi^{\prime \prime}(t)\right) \psi d x
$$

$\forall \psi \in L^{2}(\Omega)$,

the feasible test function $\psi=W^{\prime}(t)-W^{\prime \prime}(t)$ and applying Cauchy's inequality, we get the estimate

$$
\frac{d}{d t}\left\|W^{\prime}-W^{\prime \prime}\right\|_{L^{2}(\Omega)}^{2} \leqslant(2 \varepsilon+\varepsilon \kappa)\left\|W^{\prime}-W^{\prime \prime}\right\|_{L^{2}(\Omega)}^{2}+\varepsilon \kappa\left\|\Phi^{\prime}-\Phi^{\prime \prime}\right\|_{L^{2}(\Omega)}^{2} .
$$

- Step 3. The estimates for the differences $\left\|\Phi^{\prime}-\Phi^{\prime \prime}\right\|_{L^{\infty}\left[(0, T), L^{2}(\Omega)\right.}^{2},\left\|W^{\prime}-W^{\prime \prime}\right\|_{L^{2}\left[(0, T), L^{2}(\Omega)\right]}^{2}$ and $\left\|W^{\prime}-W^{\prime \prime}\right\|_{L^{\infty}\left[(0, T), L^{2}(\Omega)\right.}^{2}$. After equalization of the constants on the right-hand sides, the inequalities (5.22) and (5.24) yield together

$$
\begin{aligned}
\frac{d}{d t}\left(\left\|\Phi^{\prime}-\Phi^{\prime \prime}\right\|_{L^{2}(\Omega)}^{2}+\left\|W^{\prime}-W^{\prime \prime}\right\|_{L^{2}(\Omega)}^{2}\right) & \leqslant C \cdot\left(\left\|\Phi^{\prime}-\Phi^{\prime \prime}\right\|_{L^{2}(\Omega)}^{2}+\left\|W^{\prime}-W^{\prime \prime}\right\|_{L^{2}(\Omega)}^{2}\right) \\
+ & \left.\frac{C}{\varepsilon_{1}}\left(\left\|I_{i}^{\prime}(t)-I_{i}^{\prime \prime}(t)\right\|_{\left(W^{1,2}(\Omega)\right.}^{2}\right)^{*}+\left\|I_{e}^{\prime}(t)-I_{e}^{\prime \prime}(t)\right\|_{\left(W^{1,2}(\Omega)\right)^{*}}^{2}\right),
\end{aligned}
$$

and Gronwall's inequality finally implies that

$$
\begin{aligned}
& \left\|\Phi^{\prime}(t)-\Phi^{\prime \prime}(t)\right\|_{L^{2}(\Omega)}^{2}+\left\|W^{\prime}(t)-W^{\prime \prime}(t)\right\|_{L^{2}(\Omega)}^{2} \leqslant e^{C t}\left(\left\|\Phi^{\prime}(0)-\Phi^{\prime \prime}(0)\right\|_{L^{2}(\Omega)}^{2}\right. \\
& +\left\|W^{\prime}(0)-W^{\prime \prime}(0)\right\|_{L^{2}(\Omega)}^{2}+\frac{C}{\varepsilon_{1}} \int_{0}^{t}\left(\left\|I_{i}{ }^{\prime}(\tau)-I_{i}{ }^{\prime \prime}(\tau)\right\|_{\left(W^{1,2}(\Omega)\right)^{*}}^{2}+\left\|I_{e}{ }^{\prime}(\tau)-I_{e}{ }^{\prime \prime}(\tau)\right\|_{\left.\left.\left(W^{1,2}(\Omega)\right)^{*}\right) d \tau\right)}^{2}\right. \\
& \leqslant e^{C T} \frac{C}{\varepsilon_{1}}\left(\left\|I_{i}{ }^{\prime}-I_{i}^{\prime \prime}\right\|_{L^{2}\left[(0, T),\left(W^{1,2}(\Omega)\right)^{*}\right]}^{2}+\left\|I_{e}{ }^{\prime}-I_{e}^{\prime \prime}\right\|_{L^{2}\left[(0, T),\left(W^{1,2}(\Omega)\right)^{*}\right]}^{2}\right) \text {. }
\end{aligned}
$$

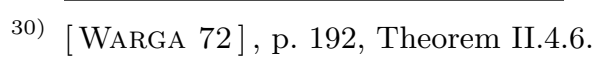


From the last inequality, we get the following estimates:

$$
\begin{aligned}
& \left\|\Phi^{\prime}-\Phi^{\prime \prime}\right\|_{L^{\infty}\left[(0, T), L^{2}(\Omega)\right.}^{2} \leqslant e^{C T} \frac{C}{\varepsilon_{1}}\left(\left\|I_{i}{ }^{\prime}-I_{i}^{\prime \prime}\right\|_{L^{2}}^{2}\left[(0, T),\left(W^{1,2}(\Omega)\right)^{*}\right]\right. \\
& +\left\|I_{e}{ }^{\prime}-I_{e}^{\prime \prime}\right\|_{\left.L^{2}\left[(0, T),\left(W^{1,2}(\Omega)\right)^{*}\right]\right)}^{2} \\
& \left.\left\|W^{\prime}-W^{\prime \prime}\right\|_{L^{\infty}\left[(0, T), L^{2}(\Omega)\right.}^{2}\right] \leqslant e^{C T} \frac{C}{\varepsilon_{1}}\left(\left\|I_{i}{ }^{\prime}-I_{i}{ }^{\prime \prime}\right\|_{L^{2}\left[(0, T),\left(W^{1,2}(\Omega)\right)^{*}\right]}^{2}\right. \\
& +\left\|I_{e}{ }^{\prime}-I_{e}^{\prime \prime}\right\|_{\left.L^{2}\left[(0, T),\left(W^{1,2}(\Omega)\right)^{*}\right]\right)}^{2} \\
& \left\|W^{\prime}-W^{\prime \prime}\right\|_{L^{2}\left[(0, T), L^{2}(\Omega)\right]}^{2} \leqslant T e^{C T} \frac{C}{\varepsilon_{1}}\left(\left\|I_{i}{ }^{\prime}-I_{i}^{\prime \prime}\right\|_{L^{2}\left[(0, T),\left(W^{1,2}(\Omega)\right)^{*}\right]}^{2}\right. \\
& \left.+\left\|I_{e}{ }^{\prime}-I_{e}{ }^{\prime \prime}\right\|_{L^{2}\left[(0, T),\left(W^{1,2}(\Omega)\right)^{*}\right]}^{2}\right) \text {. }
\end{aligned}
$$

- Step 4. The estimate for the difference $\left\|\Phi^{\prime}-\Phi^{\prime \prime}\right\|_{L^{2}\left[(0, T), W^{1,2}(\Omega)\right.}^{2}$. In (5.13), the numbers $\varepsilon_{1}, \ldots$, $\varepsilon_{4}>0$ may be alternatively chosen in such a way that

$$
\begin{aligned}
\frac{d}{d t} \| & \Phi^{\prime}-\Phi^{\prime \prime}\left\|_{L^{2}(\Omega)}^{2}+\beta\right\| \Phi^{\prime}-\Phi^{\prime \prime}\left\|_{W^{1,2}(\Omega)}^{2} \leqslant\left(\frac{C}{2 \varepsilon_{2}}+\frac{1}{\varepsilon_{3}}+2 \beta\right)\right\| \Phi^{\prime}-\Phi^{\prime \prime} \|_{L^{2}(\Omega)}^{2} \\
& \left.+\frac{1}{\varepsilon_{4}}\left\|W^{\prime}-W^{\prime \prime}\right\|_{L^{2}(\Omega)}^{2}+\frac{C}{\varepsilon_{1}}\left(\left\|I_{i}{ }^{\prime}(t)-I_{i}^{\prime \prime}(t)\right\|_{\left(W^{1,2}(\Omega)\right.}^{2}\right)^{*}+\left\|I_{e}^{\prime}(t)-I_{e}^{\prime \prime}(t)\right\|_{\left(W^{1,2}(\Omega)\right)^{*}}^{2}\right) .
\end{aligned}
$$

This implies the following modification of (5.25):

$$
\begin{aligned}
& \frac{d}{d t}\left(\left\|\Phi^{\prime}-\Phi^{\prime \prime}\right\|_{L^{2}(\Omega)}^{2}+\left\|W^{\prime}-W^{\prime \prime}\right\|_{L^{2}(\Omega)}^{2}\right)+\beta\left\|\Phi^{\prime}-\Phi^{\prime \prime}\right\|_{W^{1,2}(\Omega)}^{2} \leqslant C \cdot\left(\left\|\Phi^{\prime}-\Phi^{\prime \prime}\right\|_{L^{2}(\Omega)}^{2}\right. \\
& \left.\quad+\left\|W^{\prime}-W^{\prime \prime}\right\|_{L^{2}(\Omega)}^{2}\right)+\frac{C}{\varepsilon_{1}}\left(\left\|I_{i}{ }^{\prime}(t)-I_{i}^{\prime \prime}(t)\right\|_{\left(W^{1,2}(\Omega)\right)^{*}}^{2}+\left\|I_{e}{ }^{\prime}(t)-I_{e}^{\prime \prime}(t)\right\|_{\left(W^{1,2}(\Omega)\right)^{*}}^{2}\right) .
\end{aligned}
$$

Together with (5.27), we arrive at:

$$
\begin{gathered}
\left\|\Phi^{\prime}(t)-\Phi^{\prime \prime}(t)\right\|_{W^{1,2}(\Omega)}^{2} \leqslant C\left(\left\|I_{i}{ }^{\prime}-I_{i}^{\prime \prime}\right\|_{L^{2}\left[(0, T),\left(W^{1,2}(\Omega)\right)^{*}\right]}^{2}+\left\|I_{e}{ }^{\prime}-I_{e}{ }^{\prime \prime}\right\|_{L^{2}\left[(0, T),\left(W^{1,2}(\Omega)\right)^{*}\right]}^{2}\right) \\
\left\|\Phi^{\prime}-\Phi^{\prime \prime}\right\|_{L^{2}\left[(0, T), W^{1,2}(\Omega)\right]}^{2} \leqslant C\left(\left\|I_{i}{ }^{\prime}-I_{i}^{\prime \prime}\right\|_{L^{2}\left[(0, T),\left(W^{1,2}(\Omega)\right)^{*}\right]}^{2}\right. \\
\left.+\left\|I_{e}{ }^{\prime}-I_{e}^{\prime \prime}\right\|_{\left.L^{2}\left[(0, T),\left(W^{1,2}(\Omega)\right)^{*}\right]\right)}^{2}\right)
\end{gathered}
$$

- Step 5. The estimate for the difference $\left\|W^{\prime}-W^{\prime \prime}\right\|_{W^{1,2}\left[(0, T), L^{2}(\Omega)\right.}^{2}$. Into equation $(5.23)$, we insert the test function $\psi=\left(\partial W^{\prime}(t) / \partial t\right)-\left(\partial W^{\prime \prime}(t) / \partial t\right)$, which obviously belongs to $L^{2}\left(\Omega_{T}\right)$ and is therefore admissible. Then we get with the generalized Cauchy's inequality

$$
\begin{aligned}
& \left\langle\frac{\partial W^{\prime}}{\partial t}-\frac{\partial W^{\prime \prime}}{\partial t}, \frac{\partial W^{\prime}}{\partial t}-\frac{\partial W^{\prime \prime}}{\partial t}\right\rangle=\left\|\frac{\partial W^{\prime}}{\partial t}-\frac{\partial W^{\prime \prime}}{\partial t}\right\|_{L^{2}(\Omega)}^{2} \\
& \leqslant \varepsilon \frac{\varepsilon_{5}}{2}\left\|\frac{\partial W^{\prime}}{\partial t}-\frac{\partial W^{\prime \prime}}{\partial t}\right\|_{L^{2}(\Omega)}^{2}+\frac{\varepsilon}{2 \varepsilon_{5}}\left\|W^{\prime}-W^{\prime \prime}\right\|_{L^{2}(\Omega)}^{2} \\
& \quad+\varepsilon \kappa \frac{\varepsilon_{6}}{2}\left\|\frac{\partial W^{\prime}}{\partial t}-\frac{\partial W^{\prime \prime}}{\partial t}\right\|_{L^{2}(\Omega)}^{2}+\frac{\varepsilon \kappa}{2 \varepsilon_{6}}\left\|\Phi^{\prime}-\Phi^{\prime \prime}\right\|_{L^{2}(\Omega)}^{2}
\end{aligned}
$$

for arbitrary $\varepsilon_{5}, \varepsilon_{6}>0$. Fixing the numbers $\varepsilon_{5}$ and $\varepsilon_{6}$ in such a way that $\varepsilon \varepsilon_{5}+\varepsilon \kappa \varepsilon_{6}=1$, we find together with (5.28) and (5.29):

$$
\begin{aligned}
\left\|\frac{\partial W^{\prime}}{\partial t}-\frac{\partial W^{\prime \prime}}{\partial t}\right\|_{L^{2}(\Omega)}^{2} & \leqslant \frac{\varepsilon}{\varepsilon_{5}}\left\|W^{\prime}-W^{\prime \prime}\right\|_{L^{2}(\Omega)}^{2}+\frac{\varepsilon \kappa}{\varepsilon_{6}}\left\|\Phi^{\prime}-\Phi^{\prime \prime}\right\|_{L^{2}(\Omega)}^{2} \\
& \leqslant C\left(\left\|I_{i}{ }^{\prime}-I_{i}^{\prime \prime}\right\|_{L^{2}\left[(0, T),\left(W^{1,2}(\Omega)\right)^{*}\right]}^{2}+\left\|I_{e}{ }^{\prime}-I_{e}^{\prime \prime}\right\|_{\left.L^{2}\left[(0, T),\left(W^{1,2}(\Omega)\right)^{*}\right]\right)}^{2} \Longrightarrow\right.
\end{aligned}
$$


$\left\|\frac{\partial W^{\prime}}{\partial t}-\frac{\partial W^{\prime \prime}}{\partial t}\right\|_{L^{2}\left[(0, T), L^{2}(\Omega)\right]}^{2} \leqslant C T\left(\left\|I_{i}^{\prime}-I_{i}^{\prime \prime}\right\|_{L^{2}\left[(0, T),\left(W^{1,2}(\Omega)\right)^{*}\right]}^{2}\right.$

$$
\left.+\left\|I_{e}^{\prime}-I_{e}^{\prime \prime}\right\|_{L^{2}\left[(0, T),\left(W^{1,2}(\Omega)\right)^{*}\right]}^{2}\right),
$$

and with (5.30), we get finally:

$$
\left.\left\|W^{\prime}-W^{\prime \prime}\right\|_{W^{1,2}\left[(0, T), L^{2}(\Omega)\right.}^{2} \leqslant C\left(\left\|I_{i}{ }^{\prime}-I_{i}^{\prime \prime}\right\|_{L^{2}\left[(0, T),\left(W^{1,2}(\Omega)\right)^{*}\right.}^{2}\right]+\left\|I_{e}^{\prime}-I_{e}^{\prime \prime}\right\|_{L^{2}\left[(0, T),\left(W^{1,2}(\Omega)\right)^{*}\right]}^{2}\right) .
$$

- Step 6. The estimate for the difference $\left\|\Phi^{\prime}-\Phi^{\prime \prime}\right\|_{W^{1,4 / 3}}\left[(0, T),\left(W^{1,2}(\Omega)\right)^{*}\right]$. Let $q=4 / 3$. Exploiting the definition of the dual norm, we see that

$$
\begin{aligned}
& \int_{0}^{T}\left\|\frac{\partial \Phi^{\prime}(t)}{\partial t}-\frac{\partial \Phi^{\prime \prime}(t)}{\partial t}\right\|_{\left(W^{1,2}(\Omega)\right)^{*}}^{q} d t=\int_{0}^{T} \sup _{\|\psi\|_{W^{1,2}(\Omega)}=1}\left|\left\langle\frac{\partial \Phi^{\prime}}{\partial t}-\frac{\partial \Phi^{\prime \prime}}{\partial t}, \psi\right\rangle\right|^{q} d t \\
& \leqslant C \cdot \int_{0}^{T}\left(\sup _{\ldots}\left|\left\langle\frac{1}{1+\lambda}\left(\lambda\left(I_{i}{ }^{\prime}(t)-I_{i}{ }^{\prime \prime}(t)\right)-\left(I_{e}{ }^{\prime}(t)-I_{e}{ }^{\prime \prime}(t)\right)\right), \psi\right\rangle\right|^{q}\right. \\
& +\sup _{\ldots}\left|M\left(\Phi^{\prime}-\Phi^{\prime \prime}, \psi\right)\right|^{q}+\sup _{\ldots} \mid \int_{\Omega}\left(I_{i o n}\left(\Phi^{\prime}, W^{\prime}\right)-\left.I_{i o n}\left(\Phi^{\prime \prime}, W^{\prime \prime}\right) \psi d x\right|^{q}\right) d t \quad \Longrightarrow \\
& \left.\left(\int_{0}^{T}\left\|\frac{\partial \Phi^{\prime}}{\partial t}-\frac{\partial \Phi^{\prime \prime}}{\partial t}\right\|_{\left(W^{1,2}(\Omega)\right.}^{q}\right)^{*} d t\right)^{1 / q} \\
& \leqslant C \cdot\left(\int_{0}^{T} \sup _{\ldots}\left|\left\langle\frac{1}{1+\lambda}\left(\lambda\left(I_{i}{ }^{\prime}(t)-I_{i}{ }^{\prime \prime}(t)\right)-\left(I_{e}{ }^{\prime}(t)-I_{e}{ }^{\prime \prime}(t)\right)\right), \psi\right\rangle\right|^{2} d t\right)^{1 / 2} \\
& +C \cdot\left(\int_{0}^{T} \sup \left|M\left(\Phi^{\prime}-\Phi^{\prime \prime}, \psi\right)\right|^{2} d t\right)^{1 / 2} \\
& +C \cdot\left(\int_{0}^{T}\left(\sup _{\ldots}\left\|I_{i o n}\left(\Phi^{\prime}, W^{\prime}\right)-I_{i o n}\left(\Phi^{\prime \prime}, W^{\prime \prime}\right)\right\|_{L^{q}(\Omega)} \cdot\|\psi\|_{L^{p}(\Omega)}\right)^{q} d t\right)^{1 / q} .
\end{aligned}
$$

We estimate the three terms on the right-hand side of (5.41) separately. For the first term, we get

$$
\begin{aligned}
& \sup _{\|\psi\|_{W^{1,2}(\Omega)}=1}\left|\left\langle\frac{1}{1+\lambda}\left(\lambda\left(I_{i}{ }^{\prime}(t)-I_{i}^{\prime \prime}(t)\right)-\left(I_{e}{ }^{\prime}(t)-I_{e}{ }^{\prime \prime}(t)\right)\right), \psi\right\rangle\right|^{2} \\
& \leqslant\left\|\frac{1}{1+\lambda}\left(\lambda\left(I_{i}{ }^{\prime}(t)-I_{i}^{\prime \prime}(t)\right)-\left(I_{e}{ }^{\prime}(t)-I_{e}{ }^{\prime \prime}(t)\right)\right)\right\|_{\left(W^{1,2}(\Omega)\right)^{*}}^{2} \\
& \leqslant C\left(\left\|I_{i}{ }^{\prime}(t)-I_{i}{ }^{\prime \prime}(t)\right\|_{\left(W^{1,2}(\Omega)\right)^{*}}^{2}+\left\|I_{e}{ }^{\prime}(t)-I_{e}{ }^{\prime \prime}(t)\right\|_{\left(W^{1,2}(\Omega)\right.}^{2}\right) \\
& \quad \Longrightarrow \\
& \left(\int_{0}^{T} \sup _{\cdots}\left|\left\langle\frac{1}{1+\lambda}\left(\lambda\left(I_{i}{ }^{\prime}(t)-I_{i}{ }^{\prime \prime}(t)\right)-\left(I_{e}{ }^{\prime}(t)-I_{e}{ }^{\prime \prime}(t)\right)\right), \psi\right\rangle\right|^{2} d t\right)^{1 / 2} \\
& \leqslant C\left(\left\|I_{i}{ }^{\prime}-I_{i}{ }^{\prime \prime}\right\|_{L^{2}\left[(0, T),\left(W^{1,2}(\Omega)\right)^{*}\right]}^{2}+\left\|I_{e}{ }^{\prime}-I_{e}{ }^{\prime \prime}\right\|_{L^{2}\left[(0, T),\left(W^{1,2}(\Omega)\right)^{*}\right]}^{2}\right) .
\end{aligned}
$$

For the second term, we obtain from Lemma 2.3. and (5.34):

$$
\begin{aligned}
& \left|M\left(\Phi^{\prime}-\Phi^{\prime \prime}, \psi\right)\right|^{2} \leqslant \gamma^{2}\left\|\Phi^{\prime}-\Phi^{\prime \prime}\right\|_{W^{1,2}(\Omega)}^{2} \cdot\|\psi\|_{W^{1,2}(\Omega)}^{2} \quad \Longrightarrow \\
& \quad\left(\int_{0}^{T} \sup \left|M\left(\Phi^{\prime}-\Phi^{\prime \prime}, \psi\right)\right|^{2} d t\right)^{1 / 2} \leqslant C\left\|\Phi^{\prime}-\Phi^{\prime \prime}\right\|_{L^{2}}\left[(0, T), W^{1,2}(\Omega)\right] \\
& \quad \leqslant C\left(\left\|I_{i}^{\prime}-I_{i}^{\prime \prime}\right\|_{L^{2}\left[(0, T),\left(W^{1,2}(\Omega)\right)^{*}\right]}^{2}+\left\|I_{e}{ }^{\prime}-I_{e}^{\prime \prime}\right\|_{L^{2}\left[(0, T),\left(W^{1,2}(\Omega)\right)^{*}\right]}^{2}\right) .
\end{aligned}
$$


In order to estimate the third term, we write, relying on Lemma 2.10.,

$$
\begin{aligned}
& \left\|I_{\text {ion }}\left(\Phi^{\prime}, W^{\prime}\right)-I_{\text {ion }}\left(\Phi^{\prime \prime}, W^{\prime \prime}\right)\right\|_{L^{q}(\Omega)} \\
& \leqslant\left\|b\left(\left(\Phi^{\prime}\right)^{2}+\Phi^{\prime} \Phi^{\prime \prime}+\left(\Phi^{\prime \prime}\right)^{2}-(a+1)\left(\Phi^{\prime}+\Phi^{\prime \prime}\right)+a\right)\left(\Phi^{\prime}-\Phi^{\prime \prime}\right)\right\|_{L^{q}(\Omega)} \\
& \quad+\left\|\left(\Phi^{\prime}-\Phi^{\prime \prime}\right) W^{\prime}\right\|_{L^{q}(\Omega)}+\left\|\left(W^{\prime}-W^{\prime \prime}\right) \Phi^{\prime \prime}\right\|_{L^{q}(\Omega)}=J_{1}+J_{2}+J_{3} .
\end{aligned}
$$

For $J_{1}$, we obtain with $q=4 / 3$

$$
\begin{aligned}
J_{1}= & \left(\int_{\Omega} b^{q}\left(\left(\Phi^{\prime}\right)^{2}+\Phi^{\prime} \Phi^{\prime \prime}+\left(\Phi^{\prime \prime}\right)^{2}-(a+1)\left(\Phi^{\prime}+\Phi^{\prime \prime}\right)+a\right)^{q}\left(\Phi^{\prime}-\Phi^{\prime \prime}\right)^{q} d x\right)^{1 / q} \\
& \leqslant\left(\int _ { \Omega } b ^ { 3 q / 2 } \left(\left(\Phi^{\prime}\right)^{2}+\Phi^{\prime} \Phi^{\prime \prime}+\left(\Phi^{\prime \prime}\right)^{2}\right.\right. \\
& \left.\left.-(a+1)\left(\Phi^{\prime}+\Phi^{\prime \prime}\right)+a\right)^{3 q / 2} d x\right)^{2 / 3 q}\left(\int_{\Omega}\left(\Phi^{\prime}-\Phi^{\prime \prime}\right)^{3 q}\right)^{1 / 3 q} \\
& \leqslant C\left(1+\left\|\Phi^{\prime}(t)\right\|_{L^{4}(\Omega)}^{4}+\left\|\Phi^{\prime \prime}(t)\right\|_{L^{4}(\Omega)}^{4}\right)^{2} \cdot\left\|\Phi^{\prime}-\Phi^{\prime \prime}\right\|_{W^{1,2}(\Omega)}
\end{aligned}
$$

due to the continuous imbedding $W^{1,2}(\Omega) \hookrightarrow L^{4}(\Omega)$. Further, with (5.21) we get

$$
\begin{aligned}
& J_{2}=\left(\int_{\Omega}\left(\Phi^{\prime}-\Phi^{\prime \prime}\right)^{q}\left(W^{\prime}\right)^{q} d x\right)^{1 / q} \leqslant\left(\int_{\Omega}\left(\Phi^{\prime}-\Phi^{\prime \prime}\right)^{2} d x\right)^{2 / 3 q}\left(\int_{\Omega}\left(W^{\prime}\right)^{4} d x\right)^{1 / 3 q} \\
& =\left(\left\|\Phi^{\prime}-\Phi^{\prime \prime}\right\|_{L^{2}(\Omega)}^{4 / 3} \cdot\left\|W^{\prime}\right\|_{L^{4}(\Omega)}^{4 / 3}\right)^{3 / 4}=\left\|\Phi^{\prime}-\Phi^{\prime \prime}\right\|_{L^{2}(\Omega)} \cdot\left\|W^{\prime}\right\|_{L^{4}(\Omega)} \leqslant C\left\|\Phi^{\prime}-\Phi^{\prime \prime}\right\|_{L^{2}(\Omega)} .
\end{aligned}
$$

Finally, $J_{3}$ will be estimated together with (5.18) through

$$
\begin{aligned}
& J_{3}=\left(\int_{\Omega}\left(W^{\prime}-W^{\prime \prime}\right)^{q}\left(\Phi^{\prime \prime}\right)^{q} d x\right)^{1 / q} \leqslant\left(\int_{\Omega}\left(W^{\prime}-W^{\prime \prime}\right)^{2} d x\right)^{2 / 3 q}\left(\int_{\Omega}\left(\Phi^{\prime \prime}\right)^{4} d x\right)^{1 / 3 q} \\
& =\left(\left\|W^{\prime}-W^{\prime \prime}\right\|_{L^{2}(\Omega)}^{4 / 3} \cdot\left\|\Phi^{\prime \prime}\right\|_{L^{4}(\Omega)}^{4 / 3}\right)^{3 / 4}=\left\|W^{\prime}-W^{\prime \prime}\right\|_{L^{2}(\Omega)} \cdot\left\|\Phi^{\prime \prime}\right\|_{L^{4}(\Omega)} \leqslant C\left\|W^{\prime}-W^{\prime \prime}\right\|_{L^{2}(\Omega)} .
\end{aligned}
$$

Together, the estimates (5.52), (5.54) and (5.56) imply that

$$
\begin{gathered}
\left.\left(\int_{0}^{T} \underset{\ldots}{(} \sup \left\|I_{\text {ion }}\left(\Phi^{\prime}, W^{\prime}\right)-I_{\text {ion }}\left(\Phi^{\prime \prime}, W^{\prime \prime}\right)\right\|_{L^{4 / 3}(\Omega)} \cdot\|\psi\|_{L^{4}(\Omega)}\right)^{4 / 3} d t\right)^{3 / 4} \\
\leqslant C \cdot\left(\int _ { 0 } ^ { T } \left(\left(1+\left\|\Phi^{\prime}(t)\right\|_{L^{4}(\Omega)}^{4}+\left\|\Phi^{\prime \prime}(t)\right\|_{L^{4}(\Omega)}^{4}\right)^{2} \cdot\left\|\Phi^{\prime}-\Phi^{\prime \prime}\right\|_{W^{1,2}(\Omega)}\right.\right. \\
\left.\left.\quad+\left\|\Phi^{\prime}-\Phi^{\prime \prime}\right\|_{L^{2}(\Omega)}+\left\|W^{\prime}-W^{\prime \prime}\right\|_{L^{2}(\Omega)}\right)^{4 / 3} d t\right)^{3 / 4} \\
\leqslant C \cdot\left(\int_{0}^{T}\left(1+\left\|\Phi^{\prime}(t)\right\|_{L^{4}(\Omega)}^{4}+\left\|\Phi^{\prime \prime}(t)\right\|_{L^{4}(\Omega)}^{4}\right)^{8 / 3} \cdot\left\|\Phi^{\prime}-\Phi^{\prime \prime}\right\|_{W^{1,2}(\Omega)}^{4 / 3}\right. \\
\left.\quad+\int_{0}^{T}\left\|\Phi^{\prime}-\Phi^{\prime \prime}\right\|_{W^{1,2}(\Omega)}^{4 / 3} d t+\int_{0}^{T}\left\|W^{\prime}-W^{\prime \prime}\right\|_{L^{2}(\Omega)}^{4 / 3} d t\right)^{3 / 4} .
\end{gathered}
$$

With (5.16), (5.18), (5.27) and (5.33), we find

$$
\begin{aligned}
& \left.\left(\int_{0}^{T} \underset{\cdots}{(\sup }\left\|I_{\text {ion }}\left(\Phi^{\prime}, W^{\prime}\right)-I_{\text {ion }}\left(\Phi^{\prime \prime}, W^{\prime \prime}\right)\right\|_{L^{4 / 3}(\Omega)} \cdot\|\psi\|_{L^{4}(\Omega)}\right)^{4 / 3} d t\right)^{3 / 4} \\
& \quad \leqslant 3 C \cdot\left(\int_{0}^{T}\left(\left\|I_{i}^{\prime}-I_{i}^{\prime \prime}\right\|_{L^{2}\left[(0, T),\left(W^{1,2}(\Omega)\right)^{*}\right]}^{2}+\left\|I_{e}^{\prime}-I_{e}^{\prime \prime}\right\|_{L^{2}\left[(0, T),\left(W^{1,2}(\Omega)\right)^{*}\right]}^{2}\right)^{2 / 3} d t\right)^{3 / 4}(5 . \\
& \quad \leqslant C \cdot \operatorname{Max}\left(\left\|I_{i}{ }^{\prime}-I_{i}^{\prime \prime}\right\|_{L^{2}\left[(0, T),\left(W^{1,2}(\Omega)\right)^{*}\right]},\left\|I_{e}{ }^{\prime}-I_{e}^{\prime \prime}\right\|_{L^{2}\left[(0, T),\left(W^{1,2}(\Omega)\right)^{*}\right]}\right)
\end{aligned}
$$


Summing up, we get from (5.44), (5.47) and (5.61)

$\left\|\frac{\partial \Phi^{\prime}}{\partial t}-\frac{\partial \Phi^{\prime \prime}}{\partial t}\right\|_{L^{4 / 3}\left[(0, T),\left(W^{1,2}(\Omega)\right)^{*}\right]} \leqslant C \cdot \operatorname{Max}\left(\left\|I_{i}{ }^{\prime}-I_{i}{ }^{\prime \prime}\right\|_{L^{2}\left[(0, T),\left(W^{1,2}(\Omega)\right)^{*}\right]}\right.$
$\left.\left\|I_{e}{ }^{\prime}-I_{e}{ }^{\prime \prime}\right\|_{L^{2}\left[(0, T),\left(W^{1,2}(\Omega)\right)^{*}\right]},\left\|I_{i}{ }^{\prime}-I_{i}{ }^{\prime \prime}\right\|_{L^{2}\left[(0, T),\left(W^{1,2}(\Omega)\right)^{*}\right]}^{2},\left\|I_{e}{ }^{\prime}-I_{e}{ }^{\prime \prime}\right\|_{L^{2}\left[(0, T),\left(W^{1,2}(\Omega)\right)^{*}\right]}^{2}\right)$

and, considering (5.28),

$$
\begin{aligned}
& \left\|\Phi^{\prime}-\Phi^{\prime \prime}\right\|_{W^{1,4 / 3}\left[(0, T),\left(W^{1,2}(\Omega)\right)^{*}\right]} \leqslant C \cdot \operatorname{Max}\left(\left\|I_{i}{ }^{\prime}-I_{i}{ }^{\prime \prime}\right\|_{L^{2}\left[(0, T),\left(W^{1,2}(\Omega)\right)^{*}\right]},\right. \\
& \left.\left\|I_{e}{ }^{\prime}-I_{e}{ }^{\prime \prime}\right\|_{L^{2}\left[(0, T),\left(W^{1,2}(\Omega)\right)^{*}\right]},\left\|I_{i}{ }^{\prime}-I_{i}^{\prime \prime}\right\|_{L^{2}\left[(0, T),\left(W^{1,2}(\Omega)\right)^{*}\right]}^{2},\left\|I_{e}{ }^{\prime}-I_{e}{ }^{\prime \prime}\right\|_{L^{2}\left[(0, T),\left(W^{1,2}(\Omega)\right)^{*}\right]}^{2}\right) .
\end{aligned}
$$

- Step 7. Conclusion of the proof. In order to confirm that the left-hand side in (5.28) can be replaced by $\left\|\Phi^{\prime}-\Phi^{\prime \prime}\right\|_{C^{0}\left[[0, T], L^{2}(\Omega)\right]}$, the arguments from [Bourgault/Coudière/Pierre 09], p. 478, Subsection 5.3., may be repeated. Note that, by Theorem 4.5., (5.38) implies a bound for $\left.\left\|W^{\prime}-W^{\prime \prime}\right\|_{C^{0}\left[[0, T], L^{2}(\Omega)\right.}\right]$ as well. Finally, the continous imbedding $L^{\infty}\left[(0, T),\left(W^{1,2}(\Omega)\right)^{*}\right] \hookrightarrow L^{2}\left[(0, T),\left(W^{1,2}(\Omega)\right)^{*}\right]$ yields the claimed form (3.27) and (3.28) of the estimates. The proof is complete.

Remark. If the Rogers-McCulloch model is replaced by the FitzHugh-Nagumo model, the proof can be repeated with some obvious modifications. The same is true in the case of the linearized Aliev-Panfilov model, where (5.24) and (5.35) will be replaced by

$$
\frac{d}{d t}\left(\left\|W^{\prime}-W^{\prime \prime}\right\|_{L^{2}(\Omega)}^{2}\right) \leqslant\left(C+\frac{1}{2 \varepsilon_{8}}\right)\left(\left\|\Phi^{\prime}-\Phi^{\prime \prime}\right\|_{L^{2}(\Omega)}^{2}+\left\|W^{\prime}-W^{\prime \prime}\right\|_{L^{2}(\Omega)}^{2}\right)
$$

$$
+C \varepsilon_{8}\left\|\Phi^{\prime}-\Phi^{\prime \prime}\right\|_{W^{1,2}(\Omega)}^{2}
$$

and

$$
\begin{gathered}
\left\|\frac{\partial W^{\prime}}{\partial t}-\frac{\partial W^{\prime \prime}}{\partial t}\right\|_{L^{2}(\Omega)}^{2} \leqslant \varepsilon \frac{\varepsilon_{9}}{2}\left\|\frac{\partial W^{\prime}}{\partial t}-\frac{\partial W^{\prime \prime}}{\partial t}\right\|_{L^{2}(\Omega)}^{2}+\frac{\varepsilon}{2 \varepsilon_{9}}\left\|W^{\prime}-W^{\prime \prime}\right\|_{L^{2}(\Omega)}^{2} \\
+\varepsilon \kappa(a+1) \frac{\varepsilon_{10}}{2}\left\|\frac{\partial W^{\prime}}{\partial t}-\frac{\partial W^{\prime \prime}}{\partial t}\right\|_{L^{2}(\Omega)}^{2}+\frac{\varepsilon \kappa}{2 \varepsilon_{10}}(a+1)\left\|\Phi^{\prime}-\Phi^{\prime \prime}\right\|_{L^{2}(\Omega)}^{2} \\
+C \varepsilon_{11}\left(\left\|\Phi^{\prime}\right\|_{L^{4}(\Omega)}^{4}+\left\|\Phi^{\prime \prime}\right\|_{L^{4}(\Omega)}^{4}\right)^{1 / 2}\left\|\Phi^{\prime}-\Phi^{\prime \prime}\right\|_{W^{1,2}(\Omega)}^{2}+\frac{1}{2 \varepsilon_{8}}\left\|\frac{\partial W^{\prime}}{\partial t}-\frac{\partial W^{\prime \prime}}{\partial t}\right\|_{L^{2}(\Omega)}^{2}
\end{gathered}
$$

respectively, where $\varepsilon_{8}, \ldots, \varepsilon_{11}>0$ result from further application of the generalized Cauchy's inequality.

\section{Acknowledgement.}

The present work has been supported within the Special Research Unit "Mathematical Optimization and Applications in Biomedical Sciences" (Graz) by the Austrian Science Fund.

\section{References.}

1. [Ainseba/Bendamahne/Ruiz-Baier 10] Ainseba, B.; Bendamahne, M.; Ruiz-Baier, R.: Analysis of an optimal control problem for the tridomain model in cardiac electrophysiology. EPF Lausanne 2010; EPFL-REPORT155519, 20 pp.

2. [Aliev/Panfilov 96] Aliev, R. R.; Panfilov, A. V.: A simple two-variable model of cardiac excitation. Chaos, Solitons \& Fractals 7 (1996), $293-301$ 
3. [Berger 77] Berger, M. S.: Nonlinearity and Functional Analysis. Academic Press; New York - San Francisco London 1977

4. [Boulakia/Fernández/Gerbeau/Zemzemi 08] Boulakia, M.; Fernández, M. A.; Gerbeau, J.-F.; Zemzemi, N.: A coupled system of PDEs and ODEs arising in electrocardiograms modeling. Appl. Math. Res. Express (2008), http://dx.doi.org/10.1093/amrx/abn002 (electronically published)

5. [Bourgault/Coudière/Pierre 09] Bourgault, Y.; Coudière, Y.; Pierre, C.: Existence and uniqueness of the solution for the bidomain model used in cardiac electrophysiology. Nonlinear Analysis: Real World Appl. 10 (2009), $458-482$

6. [Brandẽo/Fernández-Cara/Magalhães/Rojas-Medar 08] Brandaõ, A. J. V.; Fernández-Cara, E.; Magalhães, P. M. D.; Rojas-Medar, M. A.: Theoretical analysis and control results for the FitzHugh-Nagumo equation. Electronic J. of Differential Equations, Vol. 2008, No. 164, 1 - 20 (electronically published)

7. [Colli Franzone/Deuflhard/Erdmann/Lang/Pavarino 06] Colli Franzone, P.; Deuflhard, P.; Erdmann, B.; Lang, J.; Pavarino, L. F.: Adaptivity in space and time for reaction-diffusion systems in electrocardiology. SIAM J. Sci. Comput. 28 (2006), $942-962$

8. [Colli Franzone/SAvaré 02] Colli Franzone, P.; Savaré, G.: Degenerate evolution systems modeling the cardiac electric field at micro- and macroscopic level. In: Lorenzi, A.; Ruf, B. (Eds.): Evolution equations, semigroups and functional analysis. In memory of Brunello Terreni. Including papers from the conference held in Milano, September 27-28, 2000. Birkhäuser; Basel - Boston - Berlin 2002 (Progress in Nonlinear Differential Equations and their Applications, Vol. 50), $49-78$

9. [Dacorogna 08] Dacorogna, B.: Direct Methods in the Calculus of Variations. Springer; New York etc. 2008, 2nd ed.

10. [DubinskiJ 65] Dubinskij, Ju. А. (Дубинский, Ю. А.): Слабая сходимость в нелинейных әллиптических и параболических уравнениях. Мат. Сборник 67 (1965) 4, $609-642$

11. [Dunford/Schwartz 88] Dunford, N.; Schwartz, J. T.: Linear Operators. Part I: General Theory. WileyInterscience; New York etc. 1988

12. [Elstrodt 96] Elstrodt, J.: Maß- und Integrationstheorie. Springer; Berlin etc. 1996

13. [Evans 98] Evans, L. C.: Partial Differential Equations. American Mathematical Society; Providence 1998

14. [FitzHugh 61] FitzHugh, R.: Impulses and physiological states in theoretical models of nerve membrane. Biophysical J. 1 (1961), $445-466$

15. [Garling 07] Garling, D. J. H.: Inequalities: A Journey into Linear Analysis. Cambridge University Press; Cambridge 2007

16. [ Ioffe/Tichomirow 79] Ioffe, A. D.; Tichomirow, V. M.: Theorie der Extremalaufgaben. VEB Deutscher Verlag der Wissenschaften; Berlin 1979

17. [Ito/Kunisch 08] Ito, K.; Kunisch, K.: Lagrange Multiplier Approach to Variational Problems and Applications. SIAM; Philadelphia 2008

18. [ Ladyženskaja/Solonnikov/Ural'Ceva 88] Ladyženskaja, O. A.; Solonnikov, V. A.; Ural'ceva, N. N.: Linear and Quasi-linear Equations of Parabolic Type. American Mathematical Society; Providence 1988

19. [Muzdeka/Barbieri 05] Muzdeka, S.; Barbieri, E.: Control theory inspired considerations for the mathematical model defibrillation. In: Proceedings of the 44th IEEE Conference on Decision and Control, 2005 and 2005 European Control Conference, 7416 - 7421

20. [NAGAiah/Kunisch 11] Nagaiah, C.; Kunisch, K.: Higher order optimization and adaptive numerical solution for optimal control of monodomain equations in cardiac electrophysiology. Appl. Num. Math. 61 (2011), $53-65$

21. [Nagaiah/Kunisch/Plank 09] Nagaiah, C.; Kunisch, K.; Plank, G.: Numerical solution for optimal control of the reaction-diffusion equations in cardiac electrophysiology. To appear in: Comput. Optim. Appl.; DOI: 10.1007/s10589-009-9280-3

22. [Nagumo/Arimoto/Yoshizawa 62] Nagumo, J.; Arimoto, S.; Yoshizawa, S.: An active pulse transmission line simulating nerve axon. Proceedings of the Institute of Radio Engineers 50 (1962), 2061 - 2070

23. [PAo 92] Pao, C. V.: Nonlinear Parabolic and Elliptic Equations. Plenum Press; New York - London 1992 
24. [Rogers/McCulloch 94] Rogers, J. M.; McCulloch, A. D.: A collocation-Galerkin finite element model of cardiac action potential propagation. IEEE Trans. Biomed. Eng. 41 (1994), $743-757$

25. [Rolewicz 76] Rolewicz, S.: Funktionalanalysis und Steuerungstheorie. Springer; Berlin - Heidelberg - New York 1976

26. [Sundnes/Lines/Cai/Nielsen/Mardal/Tveito 06] Sundnes, J.; Lines, G. T.; Cai, X.; Nielsen, B. F.; Mardal, K.-A.; Tveito, A.: Computing the Electrical Activity in the Heart. Springer; Berlin etc. 2006

27. [ Tröltzsch 09] Tröltzsch, F.: Optimale Steuerung partieller Differentialgleichungen. Vieweg + Teubner - GWV Fachverlage GmbH; Wiesbaden 2009. 2nd ed.

28. [Tung 78] Tung, L.: A Bi-Domain Model for Describing Ischemic Myocardial D-C Potentials. PhD thesis. Massachusetts Institute of Technology 1978

29. [Veneroni 06] Veneroni, M.: Reaction-diffusion systems for the microscopic cellular model of the cardiac electric field. Math. Meth. Appl. Sci. 29 (2006), 1631 - 1661

30. [Veneroni 09] Veneroni, M.: Reaction-diffusion systems for the macroscopic bidomain model of the cardiac electric field. Nonlinear Analysis: Real World Applications 10 (2009), 849 - 868

31. [Vigmond/Aguel/Trayanova 02] Vigmond, E. J.; Aguel, F.; Trayanova, N. A.: Computational techniques for solving the bidomain equations in three dimensions. IEEE Trans. Biomed. Eng. 49 (2002), 1260 - 1269

32. [WAGNER 06] Wagner, M.: Mehrdimensionale Steuerungsprobleme mit quasikonvexen Integranden. Habilitation thesis. BTU Cottbus 2006

33. [Wagner 09] Wagner, M.: Pontryagin's maximum principle for multidimensional control problems in image processing. J. Optim. Theory Appl. 140 (2009), 543 - 576

34. [WArga 72] Warga, J.: Optimal Control of Differential and Functional Equations. Academic Press; New York London 1972

35. [Weber dos Santos/Plank/Bauer/Vigmond 04] Weber dos Santos, R.; Plank, G.; Bauer, S.; Vigmond, E. J.: Parallel multigrid preconditioner for the cardiac bidomain model. IEEE Trans. Biomed. Eng. 51 (2004), $1960-1968$

36. [Yosida 95] Yosida, K.: Functional Analysis. Springer; Berlin etc. 1995 (reprint of the 6th ed. from 1980)

Last modification: 31.08 .2011

Authors' addresses / e-mail. Karl Kunisch: University of Graz, Institute for Mathematics and Scientific Computing, Heinrichstraße 36, A-8010 Graz, Austria. e-mail: karl.kunisch @ uni-graz.at

Marcus Wagner: University of Leipzig, Department of Mathematics, P. O. B. 1009 20, D-04009 Leipzig, Germany. Homepage / e-mail: www.thecitytocome.de / marcus. wagner @ math.uni-leipzig.de 